\title{
Instrumental Neutron Activation for Analysis of some Human Renal stone samples
}

\author{
Reham A Mohamed. Fatma H. El. Sweify ${ }^{a}$, Mohamed Ali ${ }^{a}$, Wafaa S. Hegazy ${ }^{b}$, \\ a) Radioisotopes production and Radiation Source Division, Atomic Energy Authority, P. O. \\ Box 3459, Cairo Egypt \\ b) University College for Girls, Arts for Science and Education, Ain Shams University, Cairo
}

Egypt

\begin{abstract}
Recently the incidence rate of renal stone disease is increased in Egypt. In addition to industrial and environmental effects in this increase, trace elements may also have a role in the formation of such stones, inspit of their significance in biological lithogenesis. In this study instrumental neutron activation analysis (INAA) technique is applied for elemental analysis of some renal stones, collected from patient living in different provinces in Egypt. The samples are collected from male, female and children. The patients are of varied ages. The analyses are carried out under various conditions of irradiation and cooling time. Major, minor and trace elements were determine. Standard material is used for accuracy and quality control measurements.
\end{abstract}

The analyzed elements were:

As, $\mathrm{Br}, \mathrm{Ca}, \mathrm{Co}, \mathrm{Cr}, \mathrm{Cu}, \mathrm{Fe}, \mathrm{Hf}, \mathrm{In}, \mathrm{Mg}, \mathrm{Mn}, \mathrm{Na}, \mathrm{Se}, \mathrm{Sr}, \mathrm{Tb}, \mathrm{V}$ and $\mathrm{Zn}$ under the experimental conditions

Some of these elements are biologicaly essential and others are dangerous or toxic.

\section{Keywords:}

Kidney stones - Renal tones - Gall stones - Neutron Activation Analysis - INAA - Short irradiation - Long irradiation.

\section{1- Introduction:}

Recently, the incidence rate of urinary and gall stones disease, in Egypt is increased. These diseases can affects the urinary tract and may lead to permanent damage of kidneys [Wijayarathna, K. S. N., \& Abeygunasekrea, A. M. 2013] as well as it may affect the function of liver. These diseases are nearly a common public health issues in population especially those living in villages. These populations represent $57.8 \%$ of all the Egyptian citizens. These diseases especially renal stones is more prevalent in Afro- Asian region [Abboud, I. A. 2008], especially those living in hot and dry climatic regions [Abeywikarama, B., Ralapanawa, U., \& Chandrajith, R. 2015].

*Corresponding Author: E-mail: romaatif33@gmail.com 
The process of formation of the stones is still not exactly understood till now. Many factors may affect the formation of these stones, among which environmental conditions, pollution resulting from industry and agriculture, dehydration and hydrogeochemical factors [Abeywikarama, B., Ralapanawa, U., \& Chandrajith, R. 2015], Drinking water hardness, dietary intake habits in particular high intake of animal proteins, oxalates and law intake of potassium bearing fruits [Tur, J., Prieto, R., \& Grases, F. 1991] and finally geographical location [ Zaravandi, A., Heidari, M., \& Mausapoor, E. 2013]

Some vegetables and fruit, in particular those contain high contents of oxalate and calcium may have essential effect on the formation of such stones.

Urinary stones are mainly biominerals which can be categorized into four groups; calcium oxalate, calcium phosphate, uric acid and strivite(magnesium ammonium phosphate) stones are the most common [Hesse, A., Tiselius, H. G., Sinener, R., \& Hoppe, B. 2009].

Calcium oxalate are usually crystalline in the forms of calcium oxalate monohydrate, dihydrate or trihydrate [Echigo, T. Kimata, M., Kyono, A., \& Simiziue, M. 2005] \& [deganello, S. 1981] and phosphate stones crystalline in one of the following form:

Calcium phosphate apatite, Calcium hydrogen phosphate dehydrates hydroxyl apatite and octa-calcium phosphate. Uric acid stones (urate) can be occur both in anhydrous and hydrated forms. Struvite is magnesium ammonium phosphate: Calcium is mostly the major constituent of urinary stones. It has high affinity towards oxalate and phosphates. Other elements are also precipitated with calcium as minor constituent like alkali and alkaline earth. Also some transition elements such as $\mathrm{Cr}, \mathrm{Mn}, \mathrm{Fe}, \mathrm{Cu}$ and $\mathrm{Zn}$ as well as $\mathrm{Pb}$ [Chandrajith et al. 2006; Zarasvandi et al. 2014; Giannossi et al. 2012]. Among urinary stones, calcium phosphate contains greater numbers of trace elements with higher concentration [Chandrajith et al. 2006; Zarasvandi et al. 2014; Giannossi et al. 2012].

In addition inorganic contents, renal stones can be occurred as mixture compounds with organic matter such as proteins, carbohydrates, lipids and organic compounds [Talham et al. 2006]. Moreover, stones crystallization can occur with the supersaturation of dissolved salts in urine [Parks et al. 2004], In view of the high incident rate of urinary stones in the south and south west of Iran a study has been carried out on trace elements content including heavy metals in 39 urinary stones. X-ray diffractometry was applied for investigation of the mineralogy of the renal stones [Abboud, I. A. 2008], [Abeywikarama, B., Ralapanawa, U., \& Chandrajith, R. 2015], Major and trace element contents were determined using ICP-MS method. Another intensive study on mineralogical, compositional and isotope characterization of human kidney stones in Sri Lanka population has been performed [Wijayarathna, K. S. N., \& Abeygunasekrea, A. M. 2013]. Other factors may have also an effect on kidney stone formation such as gender, nutrition, fluid intake, climate and even socio-economic status [Golovanova et al. 2006; Safarinejad 2007; Pourmand and Pourmand 2012; Pearle and Lotan 2012].

The prevalence of renal stone disease has been rising in both sexes, and is 2-3 times more common in males than in females [Dajani et al. 1988; Andrew and Chandru 2001; Stamatelou et al. 2003]. Peak incidence rate occurs in the second or third decade of life [Shokouhi et al. 2008]. The lifetime prevalence of kidney stone disease is estimated at 1-15 $\%$ [Pearle and Lotan 2012].

Neutron activation analysis is one of the most important analytical techniques which is used for non-destructive simultaneous multi-elements analysis of various samples of different 
chemical compositions and origins. This is attributed to its sensitivity, accuracy and specificity.

In previous work this technique has been applied for analysis of various samples for different proposes. [F. H. El-Sweify et al. (1997), F. H. El-Sweify et al. (2003), E.Metwally et al. (2004), E.A.A. El-Shazly et al. (2004). F.H. El-Sweify et al. (2007), F.H. El-Sweify et al. (2008), N.A. Tadros, F.H. El-Sweify et al. (2011), Fatma H. El-Sweify et al. (2016)].

In the present study INAA technique is applied, to analyze different human samples of renal and gall stones collected from Egyptian patients (male and female) and studying the possible factors which may influence the crystal formations in the considered organs which helps in in acquiring data concerning the role of patient's dietary intake, geographical location, and environmental conditions on the formation of such stones.

\section{2- experimental and methods:}

\subsection{Sampling:}

In this study some urinary and gall stones are collected from patients admitted Al-Matrya National Institute of kidney and urinary tract at Cairo to undergo surgery for urinary stone removal. 10 stone samples are collected from males, 5 from females and 5 from children.

Each of patient is asked to fill out a questioner prior to his/her surgery regarding personal details including gender, age, location of residence and other relevant social information are given identification (Code Numbers)

\subsubsection{Pre-treatment of stone samples:}

All samples are washed using deionized water and placed on sterile gauze, air dried, then transferred separately into polyethylene bottles. The samples are then sonicated in a water bath for $15 \mathrm{~min}$ for removing any blood clots and other remanences, after air drying, each stone is ground into powder using agate mortar and kept in a poly ethylene bottle with a code number.

\subsection{Laboratorial equipments}

\subsubsection{Compton Suppressions System with HPGe detector.}

Compton-Suppression Systems are used to reduce the background continuum for lowbackground counting. This reduction improves the overall spectrum quality especially for small volume samples. The Compton suppressions system is supplied by ORTEC. The detector, lead shielded to avoid interference for background.

\section{Characteristics of HPGe (multi channel analyzer)}

Compton-Suppression Systemsis a complete system including specially designed lead shield, timing electronics, suppression shield detector, and HPGe detector with efficiency $60 \%$, with a resolution of (2.30 keV) at FWHM and $1332 \mathrm{keV}$ and Peak to Compton 56:1

\subsubsection{Processor circulating laboratory oven.}

A laboratory oven, OF-02G, JEIO TECH manufacturing (Korea), is used for drying the bionanocomposite samples.

\subsubsection{Bi-distilled water system.}

Bi-distilled water system GFL Bi-Dist. 2104, KARL KOLB manufacturing (Germany), is used for obtaining the bi-distilled water. 


\subsection{Irradiation}

One slandered reverence material is used namely SOIL-7. Triplicate accurately weighed $(0.19$ - $0.2 \mathrm{mg}$ ) of each sample or stander is taken for irradiation. A digital analytical balance of model WH 205-4, Wiggen Hauser manufacturing (Germany), with a sensitivity of $1 \times 10^{-4} \mathrm{~g}$, is used for weighting the samples. The balance is calibrated periodically for quality control of the analysis. Very clean poly ethylene bottles and spatula are used to assure for QC of the processes.

\subsection{Gamma measuring}

Gamma ray spectra of the irradiated samples are record after cooling time ranging between 10 -20 days to allow for decay of irradiation-induced short-lived isotopes produce in the sample to avoid unnecessary radiation exposure. Analysis of other elements which produced only irradiation-induced radioactive isotopes, are analyzed under short time irradiation conditions.

\subsubsection{Gamma measurements}

\subsubsection{Relative method.}

The method is based on the simultaneous irradiation of the unknown sample with standards. The radioactivity of the standards and the samples must be measured under identical conditions using suitable measuring technique. The half-life time, and the gamma- spectra of a nuclide are the aid in establishing the identity of radioactive nuclides.

Finally, the activities of the standards and the samples are compared, and the mass of an unknown element $(\mathrm{x})$ in the sample is determined by calculation using the following equation:

$$
\frac{\text { Mass of (x)in sample }}{\text { Mass of (x)in standard }}=\frac{\text { activity from (x) in sample }}{\text { activity from (x)in standard }}
$$

There are many Standers Reference Materials (SRM), which prepared by the National Institute of Standards and Technology (NIST) and the US Geological Survey (US GS) in the United States, the International Atomic Energy Agency (IAEA) in Vienna, or other sources. These standards are often used as primary standards for NAA.

\subsubsection{Absolute method}

The induced activity due to a particular element in a sample is proportional to the amount of that element in the sample under a given set of conditions, also the knowing of the isotopic abundance is very important in carrying out sensitivity calculation and neutron flux measurement, so the activity induced in the sample is not only dependent on the amount of target element present but also on flux intensity $\mathrm{n} . \mathrm{cm}^{-2} \cdot \mathrm{Sec}^{-1}$ [J.C. Balilar, et. Al.(1973), N,N. GreenWood and A.Earnashaw et. al.(1997)]

In some cases, especially in multi-elements analysis, some elements may be present in the sample but not present in the standard. In such cases, one can make use of an analyzed element in the sample using the standard with enough certainty to analyze other elements in the sample and not present in the standard.

The possibility of using a single element as a comparator for multi-elements NAA is thus attractive. A mass (m) of an element in the sample can be determine by comparing the photopeak area (A) of a suitable gamma-line of the element's induced radioactive isotope, with that of another element $(\mathrm{A} *)$ and the mass $(\mathrm{m} *)$ determined in the sample using the standard, from the following equation [Abd EL-Razik, A. M. et. Al. (2000), Tahra El sayed Mohammed Salim (2010)] 


$$
(m)=\frac{m * \cdot A \cdot M \cdot \delta * \cdot a * \cdot b * \cdot E * \cdot S * \cdot d *}{A * \cdot M * \cdot \delta \cdot a \cdot b \cdot E \cdot S \cdot d}
$$

Where:

The asterisk $*$ refers to the element determined using the standard;

$\mathrm{M}=$ Atomic mass of the irritated element.

$\delta=$ Cross section for $(n, \gamma)$ reaction of the target nuclide.

$\mathrm{a}=$ Isotopic abundance of the target nuclide.

$\mathrm{b}=$ absolute gamma intensity in the decay scheme.

$\mathrm{E}=$ Efficiency of the detector for the $\gamma$-ray determined using standard sources.

$S=$ Saturation factor $=1-\mathrm{e}^{-\lambda t_{i}}$, where $\lambda$ is the decay constant and $t_{i}$ is the irradiation time. $\mathrm{d}=$ Decay factor $=\mathrm{e}^{-\lambda t_{\mathrm{d}}}$, where $t_{\mathrm{d}}$ is the cooling time.

In case of short irradiation time, it's important to take the dead time fractions into consecrations this is due to short irradiation, short measuring time and short half lives of the induced radioactive nuclides and the high radio activity, consequently the following equation is used for calculation.

$$
A_{0}=\frac{N_{P} \cdot\left(1+D_{t}\right) / T_{m}}{S \cdot d \cdot C \cdot w t}
$$

Where:

$\mathrm{N}_{\mathrm{P}}=$ Net peak area.

$\mathrm{D}_{\mathrm{t}}=$ dead time fraction, where dead time fraction $=$ dead time $/ 100$

$\mathrm{S}=$ Saturation factor $=1-\mathrm{e}^{-\lambda \mathrm{t}_{\mathrm{i}}}$, where $\lambda$ is the decay constant and $t_{\mathrm{i}}$ is the irradiation time.

$\mathrm{d}=$ Decay factor $=\mathrm{e}^{-\lambda \mathrm{t}_{\mathrm{d}}}$, where $\mathrm{t}_{\mathrm{d}}$ is the cooling time.

$\mathrm{C}=$ Counting factor $=\frac{1-\mathrm{e}^{-\lambda \mathrm{t}_{\mathrm{m}}}}{\lambda \mathrm{t}_{\mathrm{m}}}$, where $\mathrm{t}_{\mathrm{m}}$ is the measuring time, in case of equal measuring time counting factor will be neglected.

So final equation will be,

$$
A_{0}=\frac{N_{P} \cdot\left(1+D_{t}\right)}{S \cdot d \cdot w t}
$$

\section{3- Results and discussion}

In this chapter the data obtained from analysis of all different collected samples using the chosen analytical methods and the separation studies of different objectives are presented and discussed.

\subsection{Density measurements on stone samples.}

The densities of the collected stone samples are calculated for these purposes. The volumes of accurately weighed stone samples are measured using small accurately graduated tube with thin bottom end. The determined data are represented in the tables (1,2 and 3)

The following data are divided into three groups, namely (male, children, female). 
3.1.1. In the case of group one (males) as can be seen from the following table the stones are collected from 18 patients their ages range from (27-74) years, the density values vary between $0.6133-1.567 \mathrm{gm} / \mathrm{cm}^{3}$, except in the case of sample (48) the density range is higher $\left(2.66 \mathrm{gm} / \mathrm{cm}^{3}\right)$.

\section{- Male}

Table (1) Densities of the collected renal stones from male patients.

\begin{tabular}{|c|c|c|c|c|}
\hline ID & Age & Density $\mathbf{g} / \mathbf{c m}^{\mathbf{3}}$ & Type of stone & Location \\
\hline $\mathbf{3 0}$ & 48 & 1.4508 & Renal & Shubra AL Khimah, Al Qalyubia \\
\hline $\mathbf{3 5}$ & 50 & 1.3242 & Renal & Al Fayoum \\
\hline $\mathbf{3 6}$ & 35 & 0.6133 & Renal & Arab Al Haswah, Al Qalyubia \\
\hline $\mathbf{3 7}$ & 32 & 1.3517 & Renal & Al Qalyubia \\
\hline $\mathbf{4 5}$ & 27 & 1.2790 & Renal & Minya Al Qamh \\
\hline $\mathbf{4 8}$ & 39 & 2.66 & Renal & Helwan, Cairo Gov. \\
\hline $\mathbf{5 0}$ & 47 & 1.2244 & Renal & El Zagazig \\
\hline $\mathbf{6 8}$ & 68 & 1.4385 & Renal & Al Dokki \\
\hline $\mathbf{7 2}$ & 43 & 1.506 & Renal & Shubra Al Khema \\
\hline $\mathbf{9 5}$ & 38 & 1.08366 & Renal & Qism EL Minya \\
\hline $\mathbf{9 7}$ & 35 & 0.99866 & Renal & Al Marg \\
\hline $\mathbf{9 8}$ & 46 & 1.3603 & Renal & Shubra Al Khema \\
\hline $\mathbf{9 9}$ & 43 & 0.9021 & Renal & Imbaba \\
\hline $\mathbf{1 0 3}$ & 56 & 1.29381 & Renal & Al Ma'asarah \\
\hline $\mathbf{1 1 4}$ & 64 & 1.0837 & Renal & Al Nozha \\
\hline $\mathbf{1 1 6}$ & 74 & 1.567 & Renal & Rayoum \\
\hline $\mathbf{1 3 4}$ & 42 & 1.3491 & Renal & Renal \\
\hline $\mathbf{1 3 5}$ & 43 & 1.1751 & & \\
\hline
\end{tabular}

\subsubsection{In the case of group two (children)}

In this case Stones are collected only from four patients their ages ranging from 8-15 years. It is expected that children in this ages is difficult to find more patients suffering under this disease.

The density of the collected stones ranges from $1.23825-1.45 \mathrm{gm} / \mathrm{cm}^{3}$.

\section{- Child}

Table (2) Densities of the collected renal stones from children patients.

\begin{tabular}{|c|c|c|c|c|}
\hline ID & Age & Density $\mathbf{~ g / \mathbf { c m } ^ { 3 }}$ & Type of stone & Location \\
\hline $\mathbf{6 9}$ & 15 & 1.2775 & Renal & Rafah, North Sinai Gov. \\
\hline $\mathbf{9 0}$ & 8 & 1.45 & Renal & Al Matarya, Cairo Governorate \\
\hline $\mathbf{1 0 2}$ & 13 & 1.29381 & Renal & Al Badrashin, Giza Gov. \\
\hline $\mathbf{1 2 3}$ & 13 & 1.23825 & Renal & Al Fayoum \\
\hline
\end{tabular}

\subsubsection{In the case of group three (females)}

Stones are collected from 8 patients. Their ages ranging from (22-63) years, living in different placeless in Egypt. The density ranging from $0.1340-1.5463 \mathrm{gm} / \mathrm{cm}^{3}$, except one sample has higher density $\left(2.67 \mathrm{gm} / \mathrm{cm}^{3}\right)$ this sample ID (88) lived in Cairo 


\section{- Females}

Table (3) Densities of the collected renal stones from female patients.

\begin{tabular}{|c|c|c|c|c|}
\hline ID & Age & Density $\mathbf{g} / \mathbf{c m}^{\mathbf{3}}$ & Type of stone & Location \\
\hline $\mathbf{3 2}$ & 55 & 0.1340 & Renal & Al Omraneya \\
\hline $\mathbf{7 9}$ & 63 & 1.2754 & Renal & Al Matareya \\
\hline $\mathbf{8 2}$ & 38 & 1.5462 & Renal & Al Khankah \\
\hline $\mathbf{8 4}$ & 22 & 1.07425 & Renal & Assiut \\
\hline $\mathbf{8 6}$ & 46 & 0.9999 & Renal & Al Qalyubia \\
\hline $\mathbf{8 8}$ & 40 & 2.67 & Gall & Ghamra \\
\hline $\mathbf{1 3 0}$ & 56 & 1.4453 & Renal & Al Khanka \\
\hline $\mathbf{1 4 0}$ & 43 & 1.142838 & Renal & \\
\hline
\end{tabular}

\subsection{Effect of temperature on composition of the collected samples.}

Weighed quantities of the collected samples are taken for studying the effect of heating at $100^{\circ} \mathrm{C}$ for 24 hours and 48 hours on the change of the weight. The losses in the weight of samples after heating in each case as well as the percentage losses are determined.

The obtained data are divided into three groups, namely (male, children, and female).

3.2.1. In the case of group one (males) As can be seen from the following table the stones are collected from 18 patients their ages range from (27-74) years. The weight loss percentage after heating for $24 \mathrm{hrs}$ at $100^{\circ} \mathrm{C}$ ranges from $1.13 \%-9.41 \%$ in 8 cases and the weight loss percentage after heating for $48 \mathrm{hrs}$ at $100^{\circ} \mathrm{C}$ ranges from $(1.219 \%-5.6 \%)$ Higher percentage weight loss is observed in the case of samples (48, 68 and 96). In the case of ID 116 and 135 the weight loss percentages are, relatively very high (14.3\%) and (34\%) respectively

- Male

Table (4) weight loss and percentage weight loss values of stone samples collected from male patients after heating for $24-48 \mathrm{hrs}$ at $100^{\circ} \mathrm{C}$.

\begin{tabular}{|c|c|c|c|c|c|}
\hline ID & Age & $\begin{array}{c}\text { Weight before } \\
\text { heating }\end{array}$ & $\begin{array}{c}\text { Weight after 24 } \\
\text { h heating }\end{array}$ & Weight loss & $\begin{array}{c}\text { Weight loss } \\
\text { percentage }\end{array}$ \\
\hline $\mathbf{3 0}$ & 48 & 0.5078 & 0.4960 & 0.0118 & $9.41 \%$ \\
\hline $\mathbf{3 5}$ & 50 & 1.9862 & 1.9026 & 0.0836 & $8.36 \%$ \\
\hline $\mathbf{3 6}$ & 35 & 0.0184 & 0.01835 & $5 \times 10^{-5}$ & $0.271 \%$ \\
\hline $\mathbf{3 7}$ & 32 & 0.392 & 0.3787 & 0.0133 & $1.33 \%$ \\
\hline $\mathbf{4 5}$ & 27 & 1.4070 & 1.3911 & 0.0159 & $1.13 \%$ \\
\hline $\mathbf{4 7}$ & 59 & 1.209 & 1.0680 & 0.141 & $11.66 \%$ \\
\hline $\mathbf{4 8}$ & 39 & 0.4109 & 0.3993 & 0.0116 & $2.82 \%$ \\
\hline $\mathbf{5 0}$ & 47 & 0.6122 & 0.5744 & 0.0378 & $6.17 \%$ \\
\hline $\mathbf{6 8}$ & 68 & 1.7262 & 1.4306 & 0.2956 & $17.12 \%$ \\
\hline $\mathbf{7 2}$ & 43 & 1.3558 & 1.3177 & 0.0381 & $2.81 \%$ \\
\hline $\mathbf{9 6}$ & 46 & 0.6999 & 0.4944 & 0.2055 & $29.3 \%$ \\
\hline
\end{tabular}




\begin{tabular}{|c|c|c|c|c|c|}
\hline ID & Age & $\begin{array}{c}\text { Weight before } \\
\text { heating }\end{array}$ & $\begin{array}{c}\text { Weight after 48 } \\
\text { h heating }\end{array}$ & Weight loss & $\begin{array}{c}\text { Weight } \\
\text { percentage }\end{array}$ \\
\hline $\mathbf{9 8}$ & 46 & 0.6939 & 0.6738 & 0.0201 & $2.89 \%$ \\
\hline $\mathbf{9 9}$ & 43 & 0.1714 & 0.1651 & $6.3 \times 10^{-3}$ & $3.67 \%$ \\
\hline $\mathbf{1 0 3}$ & 56 & 1.0780 & 1.0541 & 0.0239 & $2.217 \%$ \\
\hline $\mathbf{1 1 4}$ & 64 & 0.6394 & 0.6316 & $7.8 \times 10^{-3}$ & $1.219 \%$ \\
\hline $\mathbf{1 1 6}$ & 74 & 0.4701 & 0.4027 & 0.0674 & $14.3 \%$ \\
\hline $\mathbf{1 3 4}$ & 42 & 1.2142 & 1.1461 & 0.0681 & $5.6 \%$ \\
\hline $\mathbf{1 3 5}$ & 43 & 0.9401 & 0.6178 & 0.3228 & $34 \%$ \\
\hline
\end{tabular}

\subsubsection{In the case of group two (children)}

Children studies are patients their ages ranging from 8-15 years. Children at this age supposed not to suffer from stone disease. The weight loss percentage after heating for $24 \mathrm{hrs}$ at $100^{\circ} \mathrm{C}$ in only one sample below $10 \%$ ID.90 (1.103\%), higher percentage weight loss is observed in almost cases of samples ID.123 (26.7\%), ID.102 (34\%) and ID.69 (39.54\%)

The weight loss percentage after heating for $24 \mathrm{hrs}$ at $100^{\circ} \mathrm{C}$ is indicated in the following table.

\section{- Child}

Table (5) weight loss and percentage weight loss values of stone samples collected from children patients after heating for $24 \mathrm{hrs}$ at $100^{\circ} \mathrm{C}$.

\begin{tabular}{|c|c|c|c|c|c|}
\hline ID & Age & $\begin{array}{c}\text { Weight before } \\
\text { heating at 100 }^{\circ}\end{array}$ & $\begin{array}{c}\text { Weight after 24 } \\
\text { hrs heating at } \\
\mathbf{1 0 0}^{\circ} \mathbf{C}\end{array}$ & Weight loss & $\begin{array}{c}\text { Weight loss } \\
\text { percentage }\end{array}$ \\
\hline $\mathbf{6 9}$ & 15 & 0.1533 & 0.0926 & 0.0607 & $39.54 \%$ \\
\hline $\mathbf{9 0}$ & 8 & 0.725 & 0.7170 & $8 \times 10^{-3}$ & $1.103 \%$ \\
\hline $\mathbf{1 0 2}$ & 13 & 1.4232 & 0.9370 & 0.4862 & $34 \%$ \\
\hline $\mathbf{1 2 3}$ & 13 & 0.4952 & 0.3628 & 0.1324 & $26.7 \%$ \\
\hline
\end{tabular}

In the case of group three (females)

Stones were collected from 8 patients. Their ages range from (22-63) year, living in different placeless in Egypt. These samples have weight loss percentage ranging from $(1.33 \%$ $7.45 \%)$, two samples have weight loss percentage higher $10 \%$ this sample ID.86 (12.38\%) and ID.32 (28\%)

\section{- female}

Table (6) weight loss and percentage weight loss values of stone samples collected from female patients after heating for $24-48 \mathrm{hrs}$ at $100^{\circ} \mathrm{C}$.

\begin{tabular}{|c|c|c|c|c|c|}
\hline ID & Age & $\begin{array}{c}\text { Weight before } \\
\text { heating }\end{array}$ & $\begin{array}{c}\text { Weight after 24 } \\
\text { hrs heating }\end{array}$ & Weight loss & $\begin{array}{c}\text { Weight loss } \\
\text { percentage }\end{array}$ \\
\hline $\mathbf{3 2}$ & 55 & 0.4729 & 0.3378 & 0.1351 & $28 \%$ \\
\hline $\mathbf{7 9}$ & 63 & 0.6377 & 0.6201 & 0.0176 & $2.759 \%$ \\
\hline
\end{tabular}




\begin{tabular}{|c|c|c|c|c|c|}
\hline ID & Age & $\begin{array}{c}\text { Weight before } \\
\text { heating }\end{array}$ & $\begin{array}{c}\text { Weight after 48 } \\
\text { hrs heating }\end{array}$ & Weight loss & $\begin{array}{c}\text { Weight loss } \\
\text { percentage }\end{array}$ \\
\hline $\mathbf{8 2}$ & 38 & 0.7731 & 0.7619 & 0.0112 & $1.44 \%$ \\
\hline $\mathbf{8 4}$ & 22 & 1.2891 & 1.2598 & 0.0293 & $2.27 \%$ \\
\hline $\mathbf{8 6}$ & 46 & 0.6999 & 0.6132 & 0.0867 & $12.38 \%$ \\
\hline $\mathbf{8 8}$ & 40 & 0.0574 & 0.0534 & $4.3 \times 10^{-3}$ & $7.45 \%$ \\
\hline $\mathbf{1 3 0}$ & 56 & 0.1879 & 0.1854 & $2.5 \times 10^{-3}$ & $1.33 \%$ \\
\hline $\mathbf{1 4 0}$ & 43 & 3.5429 & 3.4745 & 0.0684 & $1.93 \%$ \\
\hline
\end{tabular}

\subsection{Instrumental neutron activation analysis}

\subsubsection{Instrumental Neutron Activation Analysis under short irradiation conditions.}

The pneumatic irradiation rabbit system (PIRS) built in the vertical thermal column of the ET-RR-2 reactor (Inchass, Egypt) is used for short irradiation time measurements. Stone samples are irradiated for a period of 200 seconds. After the irradiation, each irradiation sample was transferred immediately and automatically through the pneumatic system to the counting system for $\gamma$-ray spectrometric analysis. The samples are placed at distance of about $7 \mathrm{~cm}$ from the detection system.

To obtain thermal to epithermal flux ratio a group of gold foil weighing between 0.00235 $\mathrm{mg}$ and $0.00549 \mathrm{mg}$ were sealed in polythene vials and irradiated separately at the same position.

The counting time for each sample was 200 seconds and decay time about 40-100 second. The concentration is calculated relatively by comparing concentration of standard material SOIL-7 and concentration of the same elements in stone samples.

Table(7) Relevant nuclear properties of isotopes used in INNA of trace elements in the analyzed stone samples under short irradiation and cooling times

\begin{tabular}{|c|c|c|c|c|c|c|}
\hline$\underset{t}{\text { Elemen }}$ & $\begin{array}{l}\text { Target } \\
\text { Isotope }\end{array}$ & Abundance (\%) & $\begin{array}{c}\text { Cross Section } \\
\text { (b) }\end{array}$ & $\begin{array}{c}\text { n, } \boldsymbol{\gamma} \\
\text { Produced } \\
\text { Isotope }\end{array}$ & $\boldsymbol{\gamma}$ - Lines ( Kev ) & $T_{1 / 2}$ \\
\hline I & ${ }^{127} \mathbf{I}$ & 100.0 & 6.2 & ${ }^{128} I$ & 443,527 & $25 \mathrm{~m}$ \\
\hline Mg & ${ }^{26} \mathrm{Mg}$ & 11.0 & 0.0382 & ${ }^{27} \mathrm{Mg}$ & 844,1014 & $9.46 \mathrm{~m}$ \\
\hline Mn & ${ }^{55} \mathrm{Mn}$ & 100.0 & 13.3 & ${ }^{56} \mathrm{Mn}$ & $847,1811,2113$ & $2.58 \mathrm{~h}$ \\
\hline In & ${ }^{115}$ In & 95.7 & 157 & ${ }^{116}$ In & $1294,1097,417$ & $54 \mathrm{~m}$ \\
\hline $\mathrm{Na}$ & ${ }^{23} \mathrm{Na}$ & 100.0 & 0.53 & ${ }^{24} \mathrm{Na}$ & 1369,2754 & $15 \mathrm{~h}$ \\
\hline Cl & ${ }^{37} \mathrm{Cl}$ & 24.23 & 0.428 & ${ }^{38} \mathrm{Cl}$ & 2167,1642 & $37.1 \mathrm{~m}$ \\
\hline Al & ${ }^{27} \mathrm{Al}$ & 100.0 & 0.230 & ${ }^{28} \mathrm{Al}$ & 1779 & $2.246 \mathrm{~m}$ \\
\hline $\mathbf{C a}$ & ${ }^{48} \mathrm{Ca}$ & 0.187 & 1.1 & ${ }^{49} \mathrm{Ca}$ & $\begin{array}{c}3085,4072 \\
4739\end{array}$ & $8.72 \mathrm{~m}$ \\
\hline $\mathbf{K}$ & ${ }^{41} \mathbf{K}$ & 6.7302 & 1.46 & ${ }^{42} \mathbf{K}$ & 1525 & $12.36 \mathrm{~h}$ \\
\hline $\mathbf{S i}$ & ${ }^{30} \mathrm{Si}$ & 3.10 & 0.107 & ${ }^{31} \mathbf{S i}$ & 1266 & $2.62 \mathrm{~h}$ \\
\hline $\mathbf{V}$ & ${ }^{51} \mathbf{V}$ & 99.75 & 4.88 & ${ }^{52} \mathbf{V}$ & 1434 & $3.75 \mathrm{~m}$ \\
\hline $\mathrm{Br}$ & ${ }^{79} \mathrm{Br}$ & 50.69 & 11.1 & ${ }^{80} \mathrm{Br}$ & 616,666 & $17.6 \mathrm{~m}$ \\
\hline
\end{tabular}

$\gamma$-Spectra were recorded for each sample as well as for the standard IAEA-Soil-7 the concentration of the analyzed elements was calculated reflective to that in the standard. 


\section{Concentration of the analyzed element in relation of living location as a studied factor (analyzed under short irradiation conditions)}

From the obtained results of INAA under short irradiation conditions it is indicated that $\mathrm{Na}$, $\mathrm{Mg}, \mathrm{Ca}, \mathrm{Mn}, \mathrm{Al}, \mathrm{In}$ and $\mathrm{Cl}$ are found in all analyzed stone samples in all cases male, female and children. $\mathrm{Na}, \mathrm{Mg}$ and $\mathrm{Ca}$ are present as major elements.

All are found as trace elements, the two elements $\mathrm{Cl}$ and In are also found in all analyzed stone samples, however they are analyzed qualitatively. I is analyzed nearly in all studied elements, however qualitatively. $\mathrm{Mn}$ is found as a trace element in some of the analyzed elements. $\mathrm{Si}$ is detected and determined as major elements, however in only few samples. The concentration of $\mathrm{Ca}$ and $\mathrm{Mg}$ ranging from (0.075- 74.13\%) and (0.069-47.58\%) respectively. Some samples have higher concentration of $\mathrm{Ca}$ than $\mathrm{Mg}$. The type of stones in this case is mostly calcium oxalate or calcium phosphate.

In some other stones the concentration of $\mathrm{Mg}$ is higher than calcium which may indicate that the type of stone is struvite. Some stones contain high concentration of both $\mathrm{Ca}$ and $\mathrm{Mg}$ i.e. (sample ID 35,135 ) indication that such stones contain mixed types i.e. calcium oxalate (or phosphate) + struvite.

Surprisingly, $\mathrm{Si}$ is present in seven stone samples mainly in the female stones ( 4 cases), (2 males) and (1 child) with concentration ranging (0.022-2.451\%).

Some other elements are present and determined in seven stone samples which are ID (97, 102, 123, 146, 69, 125 and 155), from these samples it is found 4 children samples, 2 males and one female.

Some other elements are found in some samples as tracers which are $\mathrm{V}, \mathrm{W}$ and $\mathrm{Cu}$

- From comparing the concentrations of $\mathrm{Ca}$ and $\mathrm{Mg}$ one may be predicts the type of stone. Thus male stone samples of ID. 30, 36, 37, 45, 46, 48, 50, 72, 98 and 116 must be calcium stones either oxalate or phosphate which is indicated from their higher Ca concentration than magnesium concentration.

On the other hand male stone samples of ID. 68, 99, 103, 114, 134 have low concentration of calcium and magnesium so it may be uric acid (urate) stones.

Only one sample of ID. 155 have higher magnesium concentration than calcium so it predicts to be sturavite stone. However, two stone samples of ID. 72 and 135 have high concentration of both calcium and magnesium must be mixed stone of calcium stones either oxalate or phosphate and sturavite stone.

- Female stone samples of high concentration of Ca than Mg are ID. 82, 88, 130 and 140 these samples consider a calcium stones either oxalate or phosphate.

Three uric acid (urate) stones defined at female samples of ID. 79, 84 and 86 which is indicate from their low $\mathrm{Ca}$ and $\mathrm{Mg}$ concentration.

One female sample of ID. 32 have higher magnesium concentration than calcium so it predicted to be sturavite stone.

- children stone samples of ID. 102 and 103 are sturavite due to high concentration of $\mathrm{Mg}$ than concentration of $\mathrm{Ca}$. only one sample of ID. 90 detect to be calcium stones either oxalate 
or phosphate, also one stone sample of ID. 146 was uric acid (urate) stone and also one stone sample of ID. 69 was a mixed stone of calcium stones either oxalate or phosphate and sturavite stone.

Table(8) Concentration of the analyzed element in relation of living location as a studied factor (analyzed under short irradiation conditions)

a- male:

\begin{tabular}{|c|c|c|c|c|c|c|c|c|c|c|c|c|c|c|c|}
\hline \multirow{2}{*}{$\begin{array}{l}\text { ID/ } \\
\text { Age }\end{array}$} & \multirow{2}{*}{ Sex } & \multicolumn{13}{|c|}{$\begin{array}{l}\text { Irradiation induced radioactive isotope } \\
\text { Concentration }(\%, \mathrm{ppm})\end{array}$} & \multirow{2}{*}{$\begin{array}{c}\text { Location } \\
\text { AL Qalyobia } \\
\text { Governorate }\end{array}$} \\
\hline & & $\begin{array}{c}{ }^{24} \mathrm{Na} \\
\%\end{array}$ & $\begin{array}{c}{ }^{27} \mathbf{M g} \\
\%\end{array}$ & $\begin{array}{l}{ }^{49} \mathrm{Ca} \\
\%\end{array}$ & ${ }^{87 m} \mathrm{Sr}$ & $\begin{array}{l}{ }^{\mathrm{s}} \mathrm{Mn} \\
\mathrm{ppm}\end{array}$ & $\begin{array}{l}{ }^{28} \mathrm{Al} \\
\mathbf{p p m}\end{array}$ & nemetn & $\begin{array}{l}{ }^{31} \mathbf{S i} \\
\%\end{array}$ & ${ }^{38} \mathrm{Cl}$ & ${ }^{80} \mathrm{Br}$ & ${ }^{128} \mathbf{I}$ & $\begin{array}{l}\text { Other } \\
\text { ppm }\end{array}$ & type & \\
\hline $37 /$ & $\mathbf{M}$ & $\begin{array}{c}0.54 \\
9\end{array}$ & $\begin{array}{c}1.03 \\
2\end{array}$ & $\begin{array}{c}42.7 \\
2\end{array}$ & - & $\begin{array}{c}0.86 \\
1\end{array}$ & $\begin{array}{c}102 . \\
7\end{array}$ & D.ND & - & D.ND & - & D.ND & - & Renal & toukh \\
\hline $\begin{array}{l}36 / \\
35 Y\end{array}$ & M & $\begin{array}{c}0.40 \\
1\end{array}$ & $\begin{array}{c}1.07 \\
1\end{array}$ & $\begin{array}{c}74.1 \\
3\end{array}$ & - & - & 380 & D.ND & - & D.ND & D.ND & D.ND & - & Renal & $\begin{array}{c}\text { Arab Al } \\
\text { Haswah, } \\
\text { Toukh }\end{array}$ \\
\hline $\begin{array}{c}95 / 38 \\
Y\end{array}$ & $\mathbf{M}$ & $\begin{array}{c}0.08 \\
8\end{array}$ & - & $\begin{array}{c}7.69 \\
1\end{array}$ & - & 1.54 & $\begin{array}{c}54.7 \\
3\end{array}$ & D.ND & $\begin{array}{c}0.49 \\
8\end{array}$ & D.ND & - & D.ND & $\begin{array}{l}{ }^{52} \mathrm{~V}=0.0 \\
18\end{array}$ & Renal & $\begin{array}{l}\text { Shubra Al } \\
\text { Khimah }\end{array}$ \\
\hline $\begin{array}{l}99 / 43 \\
Y\end{array}$ & $\mathbf{M}$ & $\begin{array}{c}0.13 \\
8\end{array}$ & - & $\begin{array}{c}0.70 \\
9\end{array}$ & - & 2.56 & $\begin{array}{c}52.7 \\
9\end{array}$ & D.ND & - & D.ND & - & D.ND & ${ }^{52} \mathrm{~V}=0.0$ & Renal & $\begin{array}{l}\text { Shubra Al } \\
\text { Khimah }\end{array}$ \\
\hline $\begin{array}{c}30 / 48 \\
Y\end{array}$ & $\mathbf{M}$ & $\begin{array}{c}1.11 \\
7\end{array}$ & $\begin{array}{c}1.17 \\
6\end{array}$ & $\begin{array}{c}42.7 \\
4\end{array}$ & D.ND & 2.21 & 86.2 & D.ND & - & D.ND & - & $\cdot$ & $\cdot$ & Renal & $\begin{array}{l}\text { Shubra Al } \\
\text { Khimah }\end{array}$ \\
\hline $\begin{array}{l}\text { ID/ } \\
\text { Age }\end{array}$ & Sex & $\begin{array}{l}{ }^{24} \mathrm{Na} \\
\%\end{array}$ & $\begin{array}{c}{ }^{27} \mathrm{Mg} \\
\%\end{array}$ & $\begin{array}{l}{ }^{49} \mathrm{Ca} \\
\%\end{array}$ & ${ }^{877} \mathrm{Sr}$ & $\begin{array}{l}{ }^{\mathrm{s} C \mathrm{Mn}} \\
\mathrm{ppm}\end{array}$ & $\begin{array}{l}{ }^{28} \mathrm{Al} \\
\mathbf{p p m}\end{array}$ & ${ }^{\mathrm{nth}} \mathrm{In}$ & $\begin{array}{l}{ }^{31} \mathbf{S i} \\
\%\end{array}$ & ${ }^{38} \mathrm{Cl}$ & ${ }^{80} \mathrm{Br}$ & ${ }^{128} \mathbf{I}$ & $\begin{array}{l}\text { Other } \\
\text { ppm }\end{array}$ & type & $\begin{array}{l}\text { AL Fayoum } \\
\text { Governorate }\end{array}$ \\
\hline $\begin{array}{c}\mathbf{3 5} / 20 \\
\mathrm{Y}\end{array}$ & $\mathbf{M}$ & $\begin{array}{c}1.52 \\
9\end{array}$ & $\begin{array}{c}15.2 \\
8\end{array}$ & $\begin{array}{c}40.2 \\
1\end{array}$ & D.ND & - & $\begin{array}{c}146 . \\
8\end{array}$ & - & - & D.ND & - & - & $\begin{array}{l}{ }^{52} \mathrm{~V}=0.0 \\
914\end{array}$ & Renal & takia \\
\hline $\begin{array}{c}72 / \\
43 Y\end{array}$ & $\mathbf{M}$ & $\begin{array}{c}0.50 \\
8\end{array}$ & $\begin{array}{c}0.14 \\
36\end{array}$ & $\begin{array}{c}39.7 \\
63\end{array}$ & - & 2.27 & $\begin{array}{c}57.1 \\
1\end{array}$ & D.ND & - & D.ND & D.ND & D.ND & - & Renal & Takia \\
\hline $\begin{array}{c}\mathbf{1 3 5} / 4 \\
3 \mathrm{Y}\end{array}$ & $\mathbf{M}$ & $\begin{array}{c}\mathbf{0 . 5 9} \\
7\end{array}$ & $\begin{array}{c}46.3 \\
3\end{array}$ & $\begin{array}{c}11.2 \\
4\end{array}$ & D.ND & - & $\begin{array}{c}67.7 \\
3\end{array}$ & D.ND & - & D.ND & - & - & $\begin{array}{c}{ }^{42} \mathbf{K}=7 . \\
79\end{array}$ & Renal & $\begin{array}{c}\text { Youssef El } \\
\text { Seddik Police } \\
\text { Station } \\
\end{array}$ \\
\hline $\begin{array}{l}\text { ID/ } \\
\text { Age }\end{array}$ & Sex & $\begin{array}{c}{ }^{24} \mathrm{Na} \\
\%\end{array}$ & $\begin{array}{c}{ }^{27} \mathrm{Mg} \\
\%\end{array}$ & $\begin{array}{c}{ }^{49} \mathrm{Ca} \\
\%\end{array}$ & ${ }^{\mathrm{s} 7 \mathrm{~m}} \mathrm{Sr}$ & $\begin{array}{l}{ }^{56} \mathrm{Mn} \\
\mathrm{ppm}\end{array}$ & $\begin{array}{l}{ }^{28} \mathrm{Al} \\
\mathbf{p p m}\end{array}$ & "ntmIn & $\begin{array}{l}{ }^{31} \mathbf{S i} \\
\%\end{array}$ & ${ }^{38} \mathrm{Cl}$ & ${ }^{80} \mathrm{Br}$ & ${ }^{128} \mathbf{I}$ & $\begin{array}{l}\text { Other } \\
\text { ppm }\end{array}$ & type & $\begin{array}{l}\text { Ash Sharquia } \\
\text { Governorate }\end{array}$ \\
\hline $\begin{array}{c}45 / \\
27 \mathrm{Y}\end{array}$ & $\mathbf{M}$ & $\begin{array}{c}0.80 \\
8\end{array}$ & $\begin{array}{c}1.70 \\
3\end{array}$ & $\begin{array}{c}41.3 \\
6\end{array}$ & - & 1.8 & $\begin{array}{c}49.0 \\
8\end{array}$ & D.ND & $\cdot$ & D.ND & - & D.ND & - & Renal & Minya Al Qamh \\
\hline $\begin{array}{l}50 / \\
47 \mathrm{Y}\end{array}$ & $\mathbf{M}$ & $\begin{array}{c}0.37 \\
0\end{array}$ & $\begin{array}{c}0.33 \\
6\end{array}$ & $\begin{array}{c}46.3 \\
6\end{array}$ & - & 2.12 & $\begin{array}{l}63.3 \\
3\end{array}$ & D.ND & - & D.ND & - & - & - & Renal & Az Zagazig \\
\hline $\begin{array}{c}155 / 5 \\
0 \mathrm{Y}\end{array}$ & $\mathbf{M}$ & $\begin{array}{c}0.16 \\
9\end{array}$ & 35 & $\begin{array}{c}3.06 \\
6\end{array}$ & - & - & $\begin{array}{c}52.8 \\
7\end{array}$ & D.ND & & D.ND & - & - & $\begin{array}{c}{ }_{63.47}^{42} \mathrm{~K}=58 \\
-\end{array}$ & Renal & $\begin{array}{l}\text { Mashtoul as } \\
\text { Souq }\end{array}$ \\
\hline $\begin{array}{l}\text { ID/ } \\
\text { Age }\end{array}$ & Sex & $\begin{array}{l}{ }^{24} \mathrm{Na} \\
\%\end{array}$ & $\begin{array}{c}{ }^{27} \mathrm{Mg} \\
\%\end{array}$ & $\begin{array}{c}{ }^{49} \mathrm{Ca} \\
\%\end{array}$ & ${ }^{877} \mathrm{Sr}$ & $\begin{array}{l}{ }^{56} \mathrm{Mn} \\
\mathrm{ppm}\end{array}$ & $\begin{array}{l}{ }^{28} \mathrm{Al} \\
\mathbf{p p m}\end{array}$ & ${ }^{116}$ In & $\begin{array}{l}{ }^{31} \mathrm{Si} \\
\%\end{array}$ & ${ }^{38} \mathrm{Cl}$ & ${ }^{80} \mathrm{Br}$ & ${ }^{128} \mathbf{I}$ & $\begin{array}{l}\text { Other } \\
\text { ppm }\end{array}$ & type & $\begin{array}{c}\text { Cairo } \\
\text { Governorate }\end{array}$ \\
\hline $\begin{array}{l}103 / \\
56 Y\end{array}$ & $\mathbf{M}$ & $\begin{array}{c}0.13 \\
2\end{array}$ & - & $\begin{array}{c}0.07 \\
5\end{array}$ & - & 1.82 & 34 & D.ND & - & D.ND & - & D.ND & - & Renal & $\begin{array}{c}\text { Al Matariyah, } \\
\text { Helmeyat AZ } \\
\text { Zaytoun } \\
\end{array}$ \\
\hline $\begin{array}{l}48 / \\
39 Y\end{array}$ & $\mathbf{M}$ & $\begin{array}{c}1.17 \\
5\end{array}$ & $\begin{array}{c}2.22 \\
9\end{array}$ & $\begin{array}{c}42.6 \\
5\end{array}$ & - & - & $\begin{array}{c}201 . \\
8\end{array}$ & D.ND & & - & - & D.ND & - & Renal & Helwan \\
\hline
\end{tabular}




\begin{tabular}{|c|c|c|c|c|c|c|c|c|c|c|c|c|c|c|c|}
\hline $\begin{array}{l}98 / \\
46 Y\end{array}$ & M & $\begin{array}{c}0.35 \\
8\end{array}$ & - & $\begin{array}{c}44.1 \\
9\end{array}$ & - & - & $\begin{array}{c}63.0 \\
6\end{array}$ & - & - & D.ND & - & D.ND & $\begin{array}{c}{ }^{52} \mathrm{~V}=0.1 \\
13\end{array}$ & Renal & AL Marg \\
\hline $\begin{array}{c}134 / 4 \\
2 Y\end{array}$ & M & $\begin{array}{c}0.04 \\
5\end{array}$ & $\begin{array}{c}0.06 \\
9\end{array}$ & $\begin{array}{c}0.24 \\
4\end{array}$ & - & - & 66.2 & - & - & D.ND & - & D.ND & $\begin{array}{c}{ }^{187} \mathbf{W}=\mathbf{D} \\
\text { ND }\end{array}$ & Renal & El-Nozha \\
\hline $\begin{array}{l}\text { ID/ } \\
\text { Age }\end{array}$ & Sex & $\underset{\%}{{ }^{2} \mathrm{Na}}$ & $\begin{array}{c}{ }^{27} \mathrm{Mg} \\
\%\end{array}$ & $\begin{array}{c}{ }^{49} \mathrm{Ca} \\
\%\end{array}$ & ${ }^{87 \mathrm{~m}} \mathrm{Sr}$ & $\begin{array}{l}{ }^{56} \mathrm{Mn} \\
\text { ppm }\end{array}$ & $\begin{array}{l}{ }^{28} \mathrm{Al} \\
\mathbf{p p m}\end{array}$ & ${ }^{116}$ In & $\begin{array}{l}{ }^{31} \mathbf{S i} \\
\%\end{array}$ & ${ }^{38} \mathrm{Cl}$ & ${ }^{80} \mathrm{Br}$ & ${ }^{128} \mathbf{I}$ & $\begin{array}{l}\text { Other } \\
\text { ppm }\end{array}$ & type & $\begin{array}{c}\text { Giza } \\
\text { Governorate }\end{array}$ \\
\hline $\begin{array}{c}114 / 6 \\
4 Y\end{array}$ & M & $\begin{array}{c}0.04 \\
9\end{array}$ & - & $\begin{array}{c}0.54 \\
17\end{array}$ & - & 2.41 & $\begin{array}{c}84.0 \\
6\end{array}$ & D.ND & - & D.ND & - & D.ND & ${ }_{\text {D.ND }}^{86} \mathrm{Cu}=$ & Renal & Imbaba \\
\hline $\begin{array}{l}68 / \\
68 Y\end{array}$ & M & $\begin{array}{c}3.12 \\
9\end{array}$ & $\begin{array}{c}0.22 \\
0\end{array}$ & $\begin{array}{c}0.38 \\
2\end{array}$ & - & 1.56 & $\begin{array}{c}52.5 \\
5\end{array}$ & D.ND & $\begin{array}{c}0.52 \\
2\end{array}$ & D.ND & - & D.ND & - & Renal & Al Dokki \\
\hline $\begin{array}{l}\text { ID/ } \\
\text { Age }\end{array}$ & Sex & $\underset{\%}{2{ }^{2} \mathbf{N a}}$ & $\begin{array}{l}{ }^{27} \mathrm{Mg} \\
\%\end{array}$ & $\begin{array}{c}{ }^{49} \mathrm{Ca} \\
\%\end{array}$ & ${ }^{87} \mathrm{~m} \mathrm{Sr}$ & $\begin{array}{l}{ }^{56} \mathrm{Mn} \\
\text { ppm }\end{array}$ & $\begin{array}{l}{ }^{28} \mathrm{Al} \\
\mathbf{p p m}\end{array}$ & "1"In & $\begin{array}{l}{ }^{31} \mathbf{S i} \\
\%\end{array}$ & 要8 $\mathrm{Cl}$ & ${ }^{80} \mathrm{Br}$ & ${ }^{128} \mathbf{I}$ & $\begin{array}{l}\text { Other } \\
\text { ppm }\end{array}$ & type & $\begin{array}{l}\text { New valley } \\
\text { Governorate }\end{array}$ \\
\hline $\begin{array}{c}116 / 7 \\
4 Y\end{array}$ & M & $\begin{array}{c}0.37 \\
4\end{array}$ & $\begin{array}{c}0.27 \\
6\end{array}$ & $\begin{array}{c}39.9 \\
3\end{array}$ & - & 1.49 & $\begin{array}{c}72.9 \\
2\end{array}$ & D.ND & - & D.ND & - & D.ND & - & Renal & Dakhla Oasis \\
\hline
\end{tabular}

b- female:

\begin{tabular}{|c|c|c|c|c|c|c|c|c|c|c|c|c|c|c|c|}
\hline \multirow{2}{*}{$\begin{array}{l}\text { ID/ } \\
\text { Age }\end{array}$} & \multirow{2}{*}{ Sex } & \multicolumn{13}{|c|}{$\begin{array}{l}\text { Irradiation induced radioactive isotope } \\
\text { Concentration }(\%, \mathrm{ppm})\end{array}$} & \multirow{2}{*}{$\begin{array}{c}\text { Location } \\
\text { AL Qalyobia } \\
\text { Governorate }\end{array}$} \\
\hline & & $\stackrel{{ }_{\%}^{24} \mathrm{Na}}{\%}$ & $\begin{array}{l}{ }^{27} \mathrm{Mg} \\
\%\end{array}$ & $\begin{array}{l}{ }^{49} \mathrm{Ca} \\
\%\end{array}$ & ${ }^{87 \mathrm{~m}} \mathrm{Sr}$ & $\begin{array}{l}{ }^{56} \mathrm{Mn} \\
\mathrm{ppm}\end{array}$ & $\begin{array}{l}{ }^{28} \mathrm{Al} \\
\mathrm{ppm}\end{array}$ & Inem In & ${ }_{\%}^{31} \mathbf{S i}$ & ${ }^{38} \mathrm{Cl}$ & ${ }^{80} \mathrm{Br}$ & ${ }^{128} \mathbf{I}$ & $\begin{array}{l}\text { Other } \\
\text { ppm }\end{array}$ & type & \\
\hline $\begin{array}{l}82 / \\
38 Y\end{array}$ & $\mathbf{F e}$ & $\begin{array}{c}0.17 \\
7\end{array}$ & - & $\begin{array}{c}41.5 \\
8\end{array}$ & - & 207. & $\begin{array}{c}61.9 \\
4\end{array}$ & D.ND & & D.ND & - & D.ND & - & Renal & $\begin{array}{l}\text { Al Khankah } \\
\text { City }\end{array}$ \\
\hline $\begin{array}{c}140 / 4 \\
3 Y\end{array}$ & $\mathbf{F e}$ & $\begin{array}{c}0.13 \\
7\end{array}$ & $\begin{array}{c}0.09 \\
05\end{array}$ & $\begin{array}{c}40.2 \\
4\end{array}$ & - & 1.4 & $\begin{array}{c}77.9 \\
3\end{array}$ & D.ND & - & D.ND & & D.ND & - & Renal & $\begin{array}{l}\text { Al Khankah } \\
\text { City }\end{array}$ \\
\hline $\begin{array}{l}86 / \\
46 Y\end{array}$ & $\mathbf{F e}$ & 3.12 & 0.22 & $\begin{array}{c}0.38 \\
26\end{array}$ & - & 1.56 & $\begin{array}{c}52.5 \\
5\end{array}$ & D.ND & $\begin{array}{c}0.52 \\
2\end{array}$ & D.ND & - & D.ND & - & Renal & Qalama \\
\hline $\begin{array}{c}130 / 6 \\
5 Y\end{array}$ & $\mathrm{Fe}$ & $\begin{array}{c}0.11 \\
5\end{array}$ & - & 27.6 & & 5.39 & $\begin{array}{c}251 . \\
5\end{array}$ & D.ND & - & D.ND & - & D.ND & . & Renal & $\begin{array}{l}\text { Al Khankah } \\
\text { City }\end{array}$ \\
\hline $\begin{array}{l}\text { ID/ } \\
\text { Age }\end{array}$ & Sex & $\stackrel{24 \mathrm{Na}}{\%}$ & $\begin{array}{l}{ }^{27} \mathrm{Mg} \\
\%\end{array}$ & $\begin{array}{l}{ }^{49} \mathrm{Ca} \\
\%\end{array}$ & ${ }^{87 \mathrm{~m}} \mathrm{Sr}$ & $\begin{array}{l}{ }^{\mathrm{s}} \mathrm{Mn} \\
\mathrm{ppm}\end{array}$ & $\begin{array}{l}{ }^{28} \mathrm{Al} \\
\mathrm{ppm}\end{array}$ & ${ }^{116} \mathrm{In}$ & ${ }_{\%}^{31} \mathbf{S i}$ & ${ }^{38} \mathrm{Cl}$ & ${ }^{80} \mathrm{Br}$ & ${ }^{128} \mathbf{I}$ & $\begin{array}{l}\text { Other } \\
\text { ppm }\end{array}$ & type & $\begin{array}{l}\text { AL Fayoum } \\
\text { Governorate }\end{array}$ \\
\hline $\begin{array}{l}84 / \\
22 Y\end{array}$ & $\mathbf{F e}$ & $\begin{array}{c}0.03 \\
5\end{array}$ & - & $\begin{array}{c}0.47 \\
28\end{array}$ & - & 1.08 & $\begin{array}{c}69.7 \\
2\end{array}$ & D.ND & $\begin{array}{c}0.02 \\
2\end{array}$ & D.ND & & D.ND & - & Renal & Al Wilidiyyah \\
\hline $\begin{array}{l}\text { ID/ } \\
\text { Age }\end{array}$ & Sex & $\begin{array}{l}{ }^{24} \mathrm{Na} \\
\%\end{array}$ & $\begin{array}{l}{ }^{27} \mathrm{Mg} \\
\%\end{array}$ & ${ }^{49} \mathrm{Ca}$ & ${ }^{87 \mathrm{~m}} \mathrm{Sr}$ & $\begin{array}{l}\text { sc Mn } \\
\text { ppm }\end{array}$ & $\begin{array}{l}{ }^{28} \mathrm{Al} \\
\mathbf{p p m}\end{array}$ & ${ }^{116} \mathrm{In}$ & ${ }_{\%}^{31} \mathrm{Si}$ & ${ }^{38} \mathrm{Cl}$ & ${ }^{80} \mathrm{Br}$ & ${ }^{128} \mathbf{I}$ & $\begin{array}{l}\text { Other } \\
\text { ppm }\end{array}$ & type & $\begin{array}{c}\text { Cairo } \\
\text { Governorate }\end{array}$ \\
\hline $\begin{array}{l}79 / \\
63 Y\end{array}$ & $\mathrm{Fe}$ & $\begin{array}{c}0.19 \\
3\end{array}$ & $\begin{array}{c}0.10 \\
8\end{array}$ & $\begin{array}{c}0.85 \\
0\end{array}$ & & 2.22 & $\begin{array}{c}62.1 \\
8\end{array}$ & D.ND & $\begin{array}{c}0.56 \\
6\end{array}$ & D.ND & & D.ND & $\begin{array}{c}{ }^{42} K=18 \\
45.54\end{array}$ & Renal & Al Matariyah \\
\hline $\begin{array}{l}88 / \\
40 Y\end{array}$ & $\mathbf{F e}$ & $\begin{array}{c}0.44 \\
4\end{array}$ & $\begin{array}{c}1.10 \\
10\end{array}$ & $\begin{array}{c}44.4 \\
61\end{array}$ & - & $\begin{array}{c}0.53 \\
9\end{array}$ & 241. & D.ND & $\begin{array}{c}2.45 \\
1\end{array}$ & D.ND & - & D.ND & $\underset{\text { ND }}{187}=$. & Gall & $\begin{array}{l}\text { El zaher, } \\
\text { Ghamrah }\end{array}$ \\
\hline $\begin{array}{c}32 / \\
55 Y\end{array}$ & $\mathbf{F e}$ & $\begin{array}{c}34.0 \\
8\end{array}$ & $\begin{array}{c}34.0 \\
80\end{array}$ & $\begin{array}{c}12.0 \\
42\end{array}$ & - & - & $\begin{array}{c}71.5 \\
3\end{array}$ & D.ND & - & D.ND & - & D.ND & - & Renal & Al Omraneyah \\
\hline
\end{tabular}


c- child

\begin{tabular}{|c|c|c|c|c|c|c|c|c|c|c|c|c|c|c|c|}
\hline \multirow{2}{*}{$\begin{array}{l}\text { ID/ } \\
\text { Age }\end{array}$} & \multirow{2}{*}{ Sex } & \multicolumn{13}{|c|}{$\begin{array}{c}\text { Irradiation induced radioactive isotope } \\
\text { Concentration }(\%, \mathrm{ppm})\end{array}$} & \multirow{2}{*}{$\begin{array}{c}\text { Location } \\
\text { Cairo } \\
\text { Governorate }\end{array}$} \\
\hline & & $\begin{array}{l}{ }^{24} \mathrm{Na} \\
\%\end{array}$ & $\begin{array}{l}{ }^{27} \mathrm{Mg} \\
\%\end{array}$ & ${ }_{\%}^{49} \mathrm{Ca}$ & ${ }^{87 / m} \mathrm{Sr}$ & $\begin{array}{l}{ }^{56} \mathrm{Mn} \\
\text { ppm }\end{array}$ & $\begin{array}{l}{ }^{28} \mathrm{Al} \\
\mathbf{p p m}\end{array}$ & ${ }^{116} \mathrm{In}$ & ${ }_{\%}^{31} \mathrm{Si}$ & ${ }^{38} \mathrm{Cl}$ & ${ }^{80} \mathrm{Br}$ & ${ }^{128} \mathbf{I}$ & $\begin{array}{l}\text { Other } \\
\text { ppm }\end{array}$ & type & \\
\hline $\begin{array}{l}90 / \\
8 Y\end{array}$ & Ch. & $\begin{array}{c}0.12 \\
7\end{array}$ & - & $\begin{array}{c}9.99 \\
08\end{array}$ & - & 1.7 & $\begin{array}{c}131 . \\
8\end{array}$ & D.ND & $\begin{array}{c}1.57 \\
6\end{array}$ & D.ND & - & - & $\begin{array}{c}{ }^{52} \mathrm{~V}=0.1 \\
2\end{array}$ & Renal & Al Matariyah \\
\hline $\begin{array}{l}\text { ID/ } \\
\text { Age }\end{array}$ & Sex & $\begin{array}{l}{ }^{24} \mathrm{Na} \\
\%\end{array}$ & $\begin{array}{l}{ }^{27} \mathrm{Mg} \\
{ }_{\%}\end{array}$ & $\begin{array}{l}{ }^{49} \mathrm{Ca} \\
\%\end{array}$ & ${ }^{87 \mathrm{~m}} \mathrm{Sr}$ & $\begin{array}{l}\text { s6 Mn } \\
\text { ppm }\end{array}$ & $\begin{array}{l}{ }^{28} \mathrm{Al} \\
\mathrm{ppm}\end{array}$ & ${ }^{1116} \mathrm{In}$ & ${ }_{\%}^{31} \mathrm{Si}$ & ${ }^{38} \mathrm{Cl}$ & ${ }^{80} \mathrm{Br}$ & ${ }^{128} \mathbf{I}$ & $\begin{array}{l}\text { Other } \\
\text { ppm }\end{array}$ & type & $\begin{array}{c}\text { Giza } \\
\text { Governorate }\end{array}$ \\
\hline $\begin{array}{c}102 / 1 \\
3 Y\end{array}$ & Ch. & $\begin{array}{c}0.25 \\
0\end{array}$ & $\begin{array}{c}47.5 \\
8\end{array}$ & $\begin{array}{c}0.16 \\
9\end{array}$ & - & - & $\begin{array}{c}68.9 \\
7\end{array}$ & D.ND & - & D.ND & - & D.ND & $\underset{431}{{ }^{42} K=16}$ & Renal & Al Badrashin \\
\hline $\begin{array}{c}123 / 1 \\
3 Y\end{array}$ & Ch. & $\begin{array}{c}0.24 \\
5\end{array}$ & $\begin{array}{c}23.1 \\
1\end{array}$ & $\begin{array}{c}0.17 \\
8\end{array}$ & - & - & $\begin{array}{c}48.8 \\
1\end{array}$ & - & - & D.ND & - & D.ND & $\begin{array}{l}{ }^{42} \mathbf{K}=10 \\
\mathbf{8 3 3}\end{array}$ & Renal & $\begin{array}{l}\text { Dahshour, Al } \\
\text { Badrashin }\end{array}$ \\
\hline $\begin{array}{c}146 / 1 \\
3 Y\end{array}$ & Ch. & $\begin{array}{c}0.02 \\
4\end{array}$ & $\begin{array}{c}2.10 \\
52\end{array}$ & $\begin{array}{c}0.11 \\
50\end{array}$ & - & $\begin{array}{c}0.15 \\
5\end{array}$ & 15.8 & D.ND & & D.ND & - & D.ND & $\begin{array}{c}{ }^{42} \mathrm{~K}=666.4 \\
1 \\
{ }^{2} \mathrm{~V}=0.02 \\
68\end{array}$ & Renal & Al Badrashin \\
\hline $\begin{array}{l}\text { ID/ } \\
\text { Age }\end{array}$ & Sex & ${ }_{\%}^{24} \mathrm{Na}$ & ${ }_{\%}^{27} \mathrm{Mg}$ & ${ }^{49} \mathrm{Ca}$ & ${ }^{87 /} \mathrm{Sr}$ & $\begin{array}{l}\text { sc Mn } \\
\text { ppm }\end{array}$ & $\begin{array}{l}{ }^{28} \mathrm{Al} \\
\mathrm{ppm}\end{array}$ & ${ }^{116} \mathrm{In}$ & ${ }_{\%}^{31} \mathrm{Si}$ & ${ }^{38} \mathrm{Cl}$ & ${ }^{80} \mathrm{Br}$ & ${ }^{128} \mathbf{I}$ & $\begin{array}{l}\text { Other } \\
\text { ppm }\end{array}$ & type & $\begin{array}{l}\text { North Siniai } \\
\text { Governorate }\end{array}$ \\
\hline $\begin{array}{c}69 / \\
15 Y\end{array}$ & Ch & $\begin{array}{c}0.78 \\
3\end{array}$ & $\begin{array}{c}42.3 \\
3\end{array}$ & $\begin{array}{c}12.7 \\
5\end{array}$ & D.ND & - & $\begin{array}{c}102 . \\
05\end{array}$ & D.ND & - & D.ND & - & - & $\begin{array}{c}{ }^{42} \mathrm{~K}=52 \\
30.97\end{array}$ & Renal & Rafah \\
\hline
\end{tabular}

\subsubsection{Instrumental Neutron Activation Analysis under long irradiation conditions.}

The collected samples are also analyzed under long irradiation and cooling time to determine other elements which needs long irradiation time in order to obtain induced-irradiation radioactive isotopes. Long lived radioactive isotopes which are used for determination of their corresponding elements. Long cooling time is unnecessary radiation exposure resulting from short lived isotopes.

Since they produce no short lived radioactive isotopes these elements produced by irradiation mainly long lived. The irradiation carried out under thermal flux of $10^{3} \mathrm{~ns}^{-1} \mathrm{~cm}^{-2}$. The ratio_of thermal and epithermal neutron flux was 20.

Table (9) Relevant nuclear properties of isotopes used in INNA of trace elements in the analyzed stone samples under long irradiation and cooling times

\begin{tabular}{|c|c|c|c|c|c|c|}
\hline $\begin{array}{c}\text { Ele } \\
\text { men } \\
t\end{array}$ & $\begin{array}{l}\text { Target } \\
\text { Isotope }\end{array}$ & $\begin{array}{c}\text { Abundance } \\
(\%)\end{array}$ & $\begin{array}{l}\text { Cross Section } \\
\text { (b ) }\end{array}$ & $\begin{array}{c}\mathrm{n}, \boldsymbol{\gamma} \\
\text { Produced } \\
\text { Isotope }\end{array}$ & $\boldsymbol{\gamma}$ - Lines ( Kev ) & $\mathbf{T}_{1 / 2}$ \\
\hline $\mathrm{Cr}$ & ${ }^{50} \mathrm{Cr}$ & 4.35 & 15.9 & ${ }^{51} \mathrm{Cr}$ & 320 & $27.70 \mathrm{~d}$ \\
\hline Au & ${ }^{197} \mathrm{Au}$ & 100.0 & 98.8 & ${ }^{198} \mathrm{Au}$ & 412 & $2.6935 \mathrm{~d}$ \\
\hline Au & ${ }^{198} \mathrm{Au}$ & 1000 & 98.8 & ${ }^{199} \mathrm{Au}$ & 158,208 & $3.139 \mathrm{~d}$ \\
\hline $\mathbf{B r}$ & ${ }^{81} \mathrm{Br}$ & 49.31 & 2.69 & ${ }^{82} \mathrm{Br}$ & $776,554,619$ & $35.34 \mathrm{~h}$ \\
\hline Zn & ${ }^{64} \mathrm{Zn}$ & 48.6 & 0.78 & ${ }^{65} \mathrm{Zn}$ & 1115 & $244 \mathrm{~d}$ \\
\hline $\mathbf{C a}$ & ${ }^{46} \mathrm{Ca}$ & 0.004 & 0.7 & ${ }^{47} \mathrm{Ca}$ & $1297,808,489$ & $5.54 \mathrm{~d}$ \\
\hline Co & ${ }^{59} \mathrm{Co}$ & 100.0 & 37.0 & ${ }^{60} \mathrm{Co}$ & 1332,1173 & $5.272 \mathrm{y}$ \\
\hline Sc & ${ }^{45} \mathrm{Sc}$ & 100.0 & 26.5 & ${ }^{46} \mathrm{Sc}$ & 889,1121 & $83.82 \mathrm{~d}$ \\
\hline Hf & ${ }^{180} \mathrm{Hf}$ & 35.2 & 12.6 & ${ }^{181} \mathrm{Hf}$ & $482,133,346$ & $42.4 \mathrm{~d}$ \\
\hline Se & ${ }^{74} \mathrm{Se}$ & 0.9 & 51.8 & ${ }^{75} \mathrm{Se}$ & $265,136,280,121,401$ & $120 \mathrm{~d}$ \\
\hline $\mathbf{R b}$ & ${ }^{85} \mathbf{R b}$ & 72.17 & 0.46 & ${ }^{86} \mathbf{R b}$ & 1077 & $18.7 \mathrm{~d}$ \\
\hline
\end{tabular}




\begin{tabular}{|c|c|c|c|c|c|c|}
\hline $\mathbf{S r}$ & ${ }^{84} \mathbf{S r}$ & 0.56 & 0.81 & ${ }^{85} \mathbf{S r}$ & 514 & $54.9 \mathrm{~d}$ \\
\hline $\mathbf{K}$ & ${ }^{39} \mathbf{K}$ & 93.258 & 1.69 & ${ }^{40} \mathbf{K}$ & 1461 & $1.38 \mathrm{E} 09 \mathrm{y}$ \\
\hline $\mathbf{S b}$ & ${ }^{123} \mathbf{S b}$ & 42.7 & 4.326 & ${ }^{124} \mathbf{S b}$ & 603,1691 & $60.3 \mathrm{~d}$ \\
\hline $\mathbf{T b}$ & ${ }^{159} \mathbf{T b}$ & 100.0 & 25.5 & ${ }^{160} \mathbf{T b}$ & $879,299,966$ & $72.1 \mathrm{~d}$ \\
\hline $\mathbf{F e}$ & ${ }^{58} \mathbf{F e}$ & 0.3 & 1.15 & ${ }^{59} \mathbf{F}$ & 1099,1292 & $45.1 \mathrm{~d}$ \\
\hline $\mathbf{E u}$ & ${ }^{151} \mathbf{E u}$ & 47.8 & 9204 & ${ }^{152} \mathbf{E u}$ & 122,344 & $13.33 \mathrm{y}$ \\
\hline $\mathbf{E u}$ & ${ }^{153} \mathbf{E u}$ & 52.2 & 390 & ${ }^{154} \mathbf{E u}$ & $123,274,723,1005$ & $8.8 \mathrm{y}$ \\
\hline $\mathbf{G a}$ & ${ }^{71} \mathbf{G a}$ & 39.9 & 4.71 & ${ }^{72} \mathbf{G a}$ & $843,2202,603$ & $14.1 \mathrm{~h}$ \\
\hline $\mathbf{M n}$ & ${ }^{53} \mathbf{M n}$ & 100.0 & 13.3 & ${ }^{54} \mathbf{M n}$ & 835 & $312.5 \mathrm{~d}$ \\
\hline $\mathbf{A s}$ & ${ }^{75} \mathbf{A s}$ & 100.0 & 4.3 & ${ }^{76} \mathbf{A s}$ & $559,657,1216$ & $26.4 \mathrm{~h}$ \\
\hline $\mathbf{C u}$ & ${ }^{65} \mathbf{C u}$ & 30.38 & 2.17 & ${ }^{66} \mathbf{C u}$ & 1039,834 & $5.1 \mathrm{~m}$ \\
\hline $\mathbf{S n}$ & ${ }^{112} \mathbf{S n}$ & 1.0 & 1.15 & ${ }^{113} \mathbf{S n}$ & 225 & $115.1 \mathrm{~d}$ \\
\hline $\mathbf{N a}$ & ${ }^{24} \mathbf{N a}$ & 10 & - & ${ }^{22} \mathbf{N a}$ & 1275 & $2.619 \mathrm{y}$ \\
\hline
\end{tabular}

${ }^{40} \mathrm{~K}$ is a natural (primordial) radioactive isotope which is present in nature

$\gamma$-Spectra were recorded for each sample as well as for the standard IAEA-Soil-7 the concentration of the analyzed elements was calculated reflective to that in the standard.

From INAA of the collected stones under long irradiation conditions the following elements are analyzed quantitatively as can be seen from the following table : these elements are $\mathrm{Cr}, \mathrm{Br}, \mathrm{Se}, \mathrm{Fe}, \mathrm{Co}$, $\mathrm{Rb}, \mathrm{Tb}, \mathrm{Au}, \mathrm{Fe}$ and $\mathrm{Hf}$.

Some elements are analyzed qualitatively such as $\mathrm{Zn}$ and Sr. All these elements are present as trace elements.

The elements $\mathrm{Zn}, \mathrm{Cr}$ and Se are present as traces and determined in some stone samples. $\mathrm{Zn}$ is detected and analyzed in almost all samples. These elements are human health essential.

Table (10) Concentration of the analyzed element in relation of living location as a studied factor (analyzed under short irradiation conditions) a- male:

\begin{tabular}{|c|c|c|c|c|c|c|c|c|c|c|c|c|}
\hline \multirow{2}{*}{$\begin{array}{l}\text { ID/ } \\
\text { Age }\end{array}$} & \multirow{2}{*}{ Sex } & \multicolumn{10}{|c|}{$\begin{array}{c}\text { Irradiation induced radioactive isotope } \\
\text { Concentration (ppm) }\end{array}$} & \multirow{2}{*}{$\begin{array}{c}\text { Location } \\
\begin{array}{l}\text { AL Qalyobia } \\
\text { Governorate }\end{array}\end{array}$} \\
\hline & & ${ }_{\mathrm{ppm}}^{51} \mathrm{Cr}$ & $\begin{array}{l}{ }^{82} \mathrm{Br} \\
\mathrm{ppm}\end{array}$ & ${ }_{\mathrm{ppm}}^{{ }^{65} \mathrm{Zn}}$ & $\begin{array}{c}{ }^{160} \mathrm{~Tb} \\
\mathrm{ppm}\end{array}$ & ${ }_{\mathrm{ppm}}^{85} \mathrm{Sr}$ & ${ }_{\mathrm{ppm}}^{75} \mathrm{Se}$ & $\begin{array}{l}{ }_{\mathbf{p p m}}^{86} \mathbf{R b} \\
.\end{array}$ & $\begin{array}{l}{ }_{\mathrm{ppm}}^{59} \mathrm{Fe} \\
\text {. }\end{array}$ & ${ }_{\mathrm{ppm}}^{60} \mathrm{Co}$ & $\begin{array}{c}\text { Other } \\
\text { ppm }\end{array}$ & \\
\hline $\begin{array}{c}37 / \\
32 Y\end{array}$ & $\mathbf{M}$ & 7.47 & - & D.ND & - & D.ND & - & - & - & 0.178 & $\cdot$ & Toukh \\
\hline $\begin{array}{c}36 / \\
35 Y\end{array}$ & M & 1.439 & 13.17 & D.ND & - & - & - & - & - & - & $\begin{array}{c}{ }^{198} \mathrm{Au}= \\
07\end{array}$ & $\begin{array}{c}\text { Arab Al } \\
\text { Haswah, Toukh }\end{array}$ \\
\hline $\begin{array}{c}99 / \\
43 \mathrm{Y}\end{array}$ & $\mathbf{M}$ & 55.21 & 1.41 & D.ND & - & D.ND & 26.740 & 4.72088 & $\begin{array}{c}3.815 E- \\
7\end{array}$ & 3.48 & ${ }_{3.0}^{181} \mathbf{H f}=$ & $\begin{array}{c}\text { Shubra Al } \\
\text { Khimah }\end{array}$ \\
\hline $\begin{array}{c}30 / \\
48 Y\end{array}$ & $\mathbf{M}$ & - & - & D.ND & 11.42 & - & - & - & - & 0.0445 & - & $\begin{array}{c}\text { Shubra Al } \\
\text { Khimah }\end{array}$ \\
\hline $\begin{array}{l}\text { ID/ } \\
\text { Age }\end{array}$ & Sex & ${ }_{\%}^{51} \mathrm{Cr}$ & $\begin{array}{c}{ }^{82} \mathrm{Br} \\
\mathrm{ppm}\end{array}$ & $\begin{array}{l}{ }^{65} \mathrm{Zn} \\
\mathrm{ppm}\end{array}$ & $\begin{array}{c}{ }^{160} \mathrm{~Tb} \\
\mathrm{ppm}\end{array}$ & ${ }_{\mathrm{ppm}}^{85} \mathrm{Sr}$ & ${ }_{\mathrm{ppm}}{ }^{75} \mathrm{Se}$ & ${ }_{\mathbf{p p m}}^{86} \mathbf{R b}$ & $\underset{\mathrm{ppm}}{{ }^{59} \mathrm{Fe}}$ & ${ }_{\mathrm{ppm}}^{60} \mathrm{Co}$ & $\begin{array}{c}\text { Other } \\
\text { ppm }\end{array}$ & $\begin{array}{l}\text { AL Fayoum } \\
\text { Governorate }\end{array}$ \\
\hline $\begin{array}{l}35 / \\
20 \mathrm{Y}\end{array}$ & M & 4.81 & - & - & - & - & - & 2.07 & - & $\begin{array}{c}4.9 \mathrm{e}- \\
05\end{array}$ & ${ }_{\text {D.ND }}^{40} \mathbf{K}=$ & - \\
\hline
\end{tabular}




\begin{tabular}{|c|c|c|c|c|c|c|c|c|c|c|c|c|}
\hline $\begin{array}{l}72 / \\
43 Y\end{array}$ & M & 3.5 & - & D.ND & - & D.ND & - & - & - & $\begin{array}{c}1.73 \mathrm{e}- \\
05\end{array}$ & - & Takia \\
\hline $\begin{array}{c}135 / 4 \\
3 Y\end{array}$ & M & 7.49 & - & D.ND & - & D.ND & - & 11.9 & - & $\begin{array}{c}\text { 9.9e- } \\
06\end{array}$ & - & $\begin{array}{c}\text { Youssef El } \\
\text { Seddik Police } \\
\text { Station }\end{array}$ \\
\hline $\begin{array}{l}\text { ID/ } \\
\text { Age }\end{array}$ & Sex & $\begin{array}{l}{ }_{\mathrm{ppm}}^{51} \mathrm{Cr} \\
\end{array}$ & $\begin{array}{l}{ }^{82} \mathrm{Br} \\
\mathrm{ppm}\end{array}$ & $\begin{array}{l}{ }^{65} \mathrm{Zn} \\
\mathrm{ppm}\end{array}$ & $\begin{array}{c}{ }^{160} \mathrm{~Tb} \\
\mathrm{ppm}\end{array}$ & ${ }_{\mathrm{ppm}}^{85} \mathrm{Sr}$ & $\begin{array}{l}{ }_{\mathrm{ppm}}^{75} \mathrm{Se} \\
\mathrm{ppm}\end{array}$ & ${ }_{\mathrm{ppm}}^{86} \mathrm{Rb}$ & $\begin{array}{l}{ }_{\mathrm{ppm}}^{59} \mathrm{Fe} \\
\end{array}$ & $\begin{array}{l}{ }^{60} \mathrm{Co} \\
\text { ppm }\end{array}$ & $\begin{array}{c}\text { Other } \\
\text { ppm }\end{array}$ & $\begin{array}{c}\text { Ash Sharquia } \\
\text { Governorate }\end{array}$ \\
\hline $\begin{array}{l}45 / \\
27 Y\end{array}$ & M & 6.93 & - & D.ND & - & - & - & - & - & 0.163 & - & $\begin{array}{c}\text { Minya Al } \\
\text { Qamh }\end{array}$ \\
\hline $\begin{array}{l}50 / \\
47 Y\end{array}$ & M & 9.42 & - & D.ND & - & D.ND & - & - & - & 0.454 & ${ }^{40} \mathbf{K N D}=$ & Az Zagazig \\
\hline $\begin{array}{l}155 / 5 \\
0 Y\end{array}$ & M & 17.6 & - & $\begin{array}{c}0.05052 \\
3\end{array}$ & - & 8.48 & - & 8.48 & - & 0.106 & - & $\begin{array}{c}\text { Mashtoul as } \\
\text { Souq }\end{array}$ \\
\hline $\begin{array}{l}\text { ID/ } \\
\text { Age }\end{array}$ & Sex & ${ }_{\mathrm{ppm}}^{51} \mathrm{Cr}$ & $\begin{array}{l}{ }_{\mathrm{p2}}^{\mathrm{Br}} \\
\mathrm{ppm}\end{array}$ & $\underset{\mathrm{ppm}}{{ }^{65} \mathrm{Zn}}$ & $\begin{array}{l}{ }^{160} \mathrm{~Tb} \\
\mathrm{ppm}\end{array}$ & ${ }^{85} \mathrm{Sr}$ & ${ }_{\mathrm{ppm}}^{75} \mathrm{Se}$ & $\begin{array}{l}{ }^{86} \mathrm{Rb} \\
\mathrm{ppm}\end{array}$ & $\underset{\mathrm{ppm}}{{ }^{59} \mathrm{Fe}}$ & $\begin{array}{l}{ }^{60} \mathrm{Co} \\
\mathrm{ppm}\end{array}$ & $\begin{array}{c}\text { Other } \\
\text { ppm }\end{array}$ & $\begin{array}{c}\text { Cairo } \\
\text { Governorate }\end{array}$ \\
\hline $\begin{array}{l}103 / \\
56 Y\end{array}$ & M & 9.77 & - & D.ND & - & - & - & - & - & & - & $\begin{array}{c}\text { Al Matariyah, } \\
\text { Helmeyat AZ } \\
\text { Zaytoun }\end{array}$ \\
\hline $\begin{array}{c}134 / 4 \\
2 Y\end{array}$ & M & 5.8 & - & D.ND & - & - & 0.0444 & - & - & $\mathbf{0 . 0 5 7 7}$ & $\begin{array}{c}{ }^{181} \mathbf{H}= \\
\mathbf{0 . 1 0 1} \\
{ }^{40} \mathbf{K}= \\
\text { DND }\end{array}$ & El-Nozha \\
\hline $68 /$ & M & 6.94 & - & D.ND & - & - & 0.084 & - & - & - & ${ }^{40} \mathrm{KND}=$ & Al Dokki \\
\hline $\begin{array}{l}\text { ID/ } \\
\text { Age }\end{array}$ & Sex & ${ }_{\mathrm{ppm}}^{51} \mathrm{Cr}$ & $\begin{array}{l}{ }^{82} \mathrm{Br} \\
\mathrm{ppm}\end{array}$ & $\begin{array}{l}{ }^{65} \mathrm{Zn} \\
\mathrm{ppm}\end{array}$ & $\begin{array}{c}{ }^{160} \mathrm{~Tb} \\
\mathrm{ppm}\end{array}$ & ${ }^{85} \mathrm{Sr}$ & $\begin{array}{l}{ }^{75} \mathrm{Se} \\
\mathrm{ppm}\end{array}$ & $\begin{array}{c}{ }^{86} \mathbf{R p m} \\
\text { ppm }\end{array}$ & $\begin{array}{l}{ }_{\mathrm{ppm}}^{59} \mathrm{Fe} \\
\end{array}$ & $\begin{array}{c}{ }^{60} \mathrm{Co} \\
\text { ppm }\end{array}$ & $\begin{array}{c}\text { Other } \\
\text { ppm }\end{array}$ & $\begin{array}{l}\text { New valley } \\
\text { Governorate }\end{array}$ \\
\hline $\begin{array}{l}116 / 7 \\
4 Y\end{array}$ & M & 7.4 & - & D.ND & - & - & - & - & 77.737 & - & ${ }_{\text {DND }}{ }^{40} \mathrm{~K}=$ & Dakhla Oasis \\
\hline
\end{tabular}

b- female:

\begin{tabular}{|c|c|c|c|c|c|c|c|c|c|c|c|c|}
\hline \multirow{2}{*}{$\begin{array}{l}\text { ID/ } \\
\text { Age }\end{array}$} & \multirow{2}{*}{ Sex } & \multicolumn{10}{|c|}{$\begin{array}{c}\text { Irradiation induced radioactive isotope } \\
\text { Concentration (ppm) }\end{array}$} & \multirow{2}{*}{$\begin{array}{c}\text { Location } \\
\begin{array}{l}\text { AL Qalyobia } \\
\text { Governorate }\end{array}\end{array}$} \\
\hline & & ${ }_{\mathrm{ppm}}^{51} \mathrm{Cr}$ & $\begin{array}{l}{ }^{82} \mathrm{Br} \\
\mathrm{ppm}\end{array}$ & ${ }_{\mathrm{ppm}}^{65} \mathrm{Zn}$ & $\begin{array}{l}{ }^{160} \mathrm{~Tb} \\
\mathrm{ppm}\end{array}$ & ${ }_{\mathrm{ppm}}^{85} \mathrm{Sr}$ & ${ }_{\mathrm{ppm}}^{75} \mathrm{Se}$ & $\begin{array}{c}{ }^{86} \mathbf{R b} \\
\mathbf{p p m}\end{array}$ & $\begin{array}{l}{ }_{\mathrm{ppm}}^{59} \mathrm{Fe} \\
\text {. }\end{array}$ & ${ }_{\mathrm{ppm}}^{60} \mathrm{Co}$ & $\begin{array}{c}\text { Other } \\
\text { ppm }\end{array}$ & \\
\hline $\begin{array}{l}82 / \\
38 Y\end{array}$ & $\mathbf{F e}$ & 6.5 & - & D.ND & - & D.ND & 0.046 & - & - & - & - & $\begin{array}{c}\text { Al Khankah } \\
\text { City }\end{array}$ \\
\hline $\begin{array}{c}140 / 4 \\
3 Y\end{array}$ & $\mathrm{Fe}$ & 3.18 & - & D.ND & - & - & 0.0435 & - & - & - & - & $\begin{array}{c}\text { Al Khankah } \\
\text { City }\end{array}$ \\
\hline $\begin{array}{l}86 / \\
46 Y\end{array}$ & $\mathbf{F e}$ & 5.03 & - & D.ND & - & - & - & $\begin{array}{c}6.77 \mathrm{E}- \\
05\end{array}$ & 39.51 & 0.702 & - & Qalama \\
\hline $\begin{array}{c}130 / 6 \\
5 Y\end{array}$ & $\mathbf{F e}$ & 94.336 & 0.9304 & D.ND & - & - & - & - & $\begin{array}{c}6.734 \mathrm{E}- \\
7\end{array}$ & 0.7593 & - & $\begin{array}{c}\text { Al Khankah } \\
\text { City }\end{array}$ \\
\hline $\begin{array}{l}\text { ID/ } \\
\text { Age }\end{array}$ & Sex & ${ }_{\%}^{51} \mathrm{Cr}$ & $\begin{array}{l}{ }^{82} \mathrm{Br} \\
\mathrm{ppm}\end{array}$ & ${ }_{\mathrm{ppm}}^{65} \mathrm{Zn}$ & $\begin{array}{l}{ }^{160} \mathrm{~Tb} \\
\mathrm{ppm}\end{array}$ & ${ }_{\mathrm{ppm}}^{85} \mathrm{Sr}$ & ${ }_{\mathrm{ppm}}^{75} \mathrm{Se}$ & $\begin{array}{c}{ }_{\mathrm{ppm}}^{86} \mathbf{R b} \\
\mathrm{ppm}\end{array}$ & $\begin{array}{l}{ }_{\mathrm{ppm}}^{59} \mathrm{Fe} \\
\text {. }\end{array}$ & ${ }_{\mathrm{ppm}}^{60} \mathrm{Co}$ & $\begin{array}{c}\text { Other } \\
\text { ppm }\end{array}$ & $\begin{array}{l}\text { AL Fayoum } \\
\text { Governorate }\end{array}$ \\
\hline $\begin{array}{l}84 / \\
22 Y\end{array}$ & $\mathrm{Fe}$ & 5.46 & - & D.ND & - & - & 9431 & - & - & 0.016 & - & $\begin{array}{c}\text { Al } \\
\text { Wilidiyyah }\end{array}$ \\
\hline $\begin{array}{l}\text { ID/ } \\
\text { Age }\end{array}$ & Sex & ${ }_{\mathrm{ppm}}^{51} \mathrm{Cr}$ & $\begin{array}{l}{ }^{82} \mathrm{Br} \\
\mathrm{ppm}\end{array}$ & ${ }_{\mathrm{ppm}}^{65} \mathrm{Zn}$ & $\begin{array}{l}{ }^{160} \mathrm{~Tb} \\
\mathrm{ppm}\end{array}$ & ${ }^{85} \mathrm{Sr}$ & ${ }_{\mathrm{ppm}}^{75} \mathrm{Se}$ & ${ }_{\mathrm{ppm}}^{86} \mathbf{R b}$ & $\underset{\mathrm{ppm}}{{ }^{59} \mathrm{Fe}}$ & ${ }_{\mathrm{ppm}}^{60} \mathrm{Co}$ & $\begin{array}{c}\text { Other } \\
\text { ppm }\end{array}$ & $\begin{array}{c}\text { Cairo } \\
\text { Governorate }\end{array}$ \\
\hline $\begin{array}{l}88 / \\
40 Y\end{array}$ & $\mathbf{F e}$ & 2.943 & 1.088 & D.ND & & & 0.271 & - & - & 0.06858 & ${ }_{1.346 \mathrm{e}-7}^{198} \mathrm{Au}=$ & $\begin{array}{l}\text { El zaher, } \\
\text { Ghamrah }\end{array}$ \\
\hline
\end{tabular}




\begin{tabular}{|c|c|c|c|c|c|c|c|c|c|c|c|c|}
\hline $32 /$ & Fe & 3.93 & - & D.ND & - & - & - & 14.27 & - & 0.0831 & - & Al Omraneyah \\
\hline
\end{tabular}

C- child:

\begin{tabular}{|c|c|c|c|c|c|c|c|c|c|c|c|c|}
\hline \multirow{2}{*}{$\begin{array}{l}\text { ID/ } \\
\text { Age }\end{array}$} & \multirow{2}{*}{ Sex } & \multicolumn{10}{|c|}{$\begin{array}{c}\text { Irradiation induced radioactive isotope } \\
\text { Concentration (ppm) }\end{array}$} & \multirow{2}{*}{$\begin{array}{c}\text { Location } \\
\begin{array}{c}\text { Cairo } \\
\text { Governorate }\end{array}\end{array}$} \\
\hline & & $\begin{array}{c}{ }^{51} \mathrm{Cr} \\
\text { ppm }\end{array}$ & $\begin{array}{c}{ }^{82} \mathrm{Br} \\
\text { ppm }\end{array}$ & $\begin{array}{l}{ }^{65} \mathrm{Zn} \\
\mathrm{ppm}\end{array}$ & $\begin{array}{c}{ }^{160} \mathrm{~Tb} \\
\mathrm{ppm}\end{array}$ & ${ }^{85} \mathrm{Sr}$ & $\begin{array}{l}{ }_{\mathrm{ppm}}^{75} \mathrm{Se} \\
\mathrm{ppm}\end{array}$ & $\begin{array}{c}{ }^{86} \mathbf{R b} \\
\text { ppm }\end{array}$ & $\begin{array}{c}{ }_{\mathrm{ppm}}^{59} \mathrm{Fe} \\
\end{array}$ & $\begin{array}{c}{ }^{60} \mathrm{Co} \\
\text { ppm }\end{array}$ & $\begin{array}{c}\text { Other } \\
\text { ppm }\end{array}$ & \\
\hline $\begin{array}{l}90 / \\
8 Y\end{array}$ & Ch & 6.97 & - & D.ND & 0.0325 & - & 0.0667 & - & - & - & $\begin{array}{c}{ }^{181} \mathbf{H f}= \\
{ }_{0.0815}\end{array}$ & Al Matariyah \\
\hline $\begin{array}{l}\text { ID/ } \\
\text { Age }\end{array}$ & Sex & $\begin{array}{c}{ }^{51} \mathrm{Cr} \\
\mathrm{ppm}\end{array}$ & $\begin{array}{l}{ }^{82} \mathrm{Br} \\
\text { ppm }\end{array}$ & $\begin{array}{l}{ }_{\mathrm{ppm}}^{65} \mathrm{Zn} \\
\end{array}$ & $\begin{array}{l}{ }^{160} \mathrm{~Tb} \\
\mathrm{ppm}\end{array}$ & ${ }^{85} \mathrm{Sr}$ & ${ }_{\mathrm{ppm}}^{{ }_{\mathrm{75}} \mathrm{Se}}$ & $\begin{array}{l}{ }_{\mathrm{ppm}}^{86} \mathrm{Rb} \\
.\end{array}$ & $\begin{array}{c}{ }^{59} \mathrm{Fe} \\
\text { ppm }\end{array}$ & $\begin{array}{l}{ }^{60} \mathrm{Co} \\
\text { ppm }\end{array}$ & $\begin{array}{c}\text { Other } \\
\text { ppm }\end{array}$ & $\begin{array}{c}\text { Giza } \\
\text { Governorate }\end{array}$ \\
\hline $\begin{array}{l}102 / 1 \\
3 Y\end{array}$ & $\mathrm{Ch}$ & 12.43 & - & D.ND & - & - & - & 6.62 & - & 0.086 & - & Al Badrashin \\
\hline $\begin{array}{l}123 / 1 \\
3 Y\end{array}$ & Ch & 5.03 & - & D.ND & - & - & - & - & - & 0.15 & - & $\begin{array}{c}\text { Dahshour, Al } \\
\text { Badrashin }\end{array}$ \\
\hline $\begin{array}{l}146 / 1 \\
3 Y\end{array}$ & Ch & 1.73 & 0.149 & D.ND & - & - & - & 4.98 & - & - & - & Al Badrashin \\
\hline $\begin{array}{l}\text { ID/ } \\
\text { Age }\end{array}$ & Sex & ${ }_{\text {ppm }}^{51} \mathbf{C r}$ & $\begin{array}{l}{ }^{82} \mathrm{Br} \\
\mathrm{ppm}\end{array}$ & ${ }_{\mathrm{ppm}}^{65} \mathrm{Zn}$ & $\begin{array}{l}{ }^{160} \mathrm{~Tb} \\
\mathrm{ppm}\end{array}$ & ${ }^{85} \mathrm{Sr}$ & $\begin{array}{l}{ }_{\mathrm{ppm}}^{75} \mathrm{Se} \\
\mathrm{pp}\end{array}$ & $\begin{array}{l}{ }^{86} \mathrm{Rb} \\
\mathrm{ppm}\end{array}$ & $\underset{\mathrm{ppm}}{{ }^{59} \mathrm{Fe}}$ & $\begin{array}{l}{ }^{60} \mathrm{Co} \\
\text { ppm }\end{array}$ & $\begin{array}{c}\text { Other } \\
\text { ppm }\end{array}$ & $\begin{array}{l}\text { North Siniai } \\
\text { Governorate }\end{array}$ \\
\hline $\begin{array}{l}69 / \\
15 Y\end{array}$ & Ch & 27.04 & 0.399 & D.ND & 0.25 & D.ND & - & - & - & 0.111 & - & Rafah \\
\hline
\end{tabular}

\section{References}

Abboud, I. A." Mineralogy and chemistry of urinary stones: Patients from North Jordan". Environmental Geochemistry and Health, 30(5), 445-463. (2008). https://doi.org/10. 1007/s10653007-9128-7.

Abeywickarama, B., Ralapanawa, U., \& Chandrajith, R. "Geoenvironmental factors related to high incidence of human urinary calculi (kidney stones) in Central Highlands of Sri Lanka". Environmental Geochemistry and Health, 38(5), 1203-1214. https://doi.org/10.1007/s10653-015- 9785-x (2015).

Andrew, J. P. \& Chandru, P. S. "Diagnosis and initial management of kidney stones". American Family Physician, http://www.aatp.org/atp/20010401/1329.html. p. 17. (2001).

Abd EL-Razik, A. M. (2000).

Chandrajith, R., Wijewardana, G., Dissanayake, C. B., \& Abeygunasekara, A. "Biomineralogy of human urinary calculi (kidney stones) from some geographic regions of Sri Lanka". Environmental Geochemistry and Health, 28(4), 393-399. https://doi.org/10.1007/s10653- 006-9048-y (2006).

Dajani, A., Khadra, A. A., \& Baghdadi, F. "Urolithiasis in Jordanian Children.: A Report of 52 Cases". British Journal of Urology, 61(6), 482-486. (1988).

Deganello, S. "The structure of whewellite, $\mathrm{CaC}_{2} \mathrm{O} 4 \mathrm{H}_{2} \mathrm{O}$ at $328 \mathrm{~K}$. Acta Crystallographica Section B: Structural Crystallography and Crystal Chemistry", 37(4), 826-829. (1981). 
Echigo, T., Kimata, M., Kyono, A., \& Shimizu, M." Reinvestigation of the crystal structure of whewellite $\left[\mathrm{Ca}\left(\mathrm{C}_{2} \mathrm{O}_{4}\right) \mathrm{H}_{2} \mathrm{O}\right]$ and the dehydration mechanism of caoxite $\left[\mathrm{Ca}\left(\mathrm{C}_{2} \mathrm{O}_{4}\right)_{3} \mathrm{H}_{2} \mathrm{O}\right] "$. Mineralogical Magazine, 69(1), 77-88 (2005).

El-Shazy, E. A. A., Abo Zahra, Sh. F. El-Sweify, F. H., Kanias , G. G.: 'Simultaneous Multi-element determination in some cosmetic samples of different origin using neutron activation analysis". J. Radiochim. Acta 92,111(2004).

El-Sweify, F.H, El-Sweify, A.H.H., Abd El-Razek, A.M.: "Flax (Linumusitatissimum L.)As a source for animal feed and its contents of some elements". Proc. Conf. "Food-borne contamination and Egyptian health" J. Mansoura Univ., Egypt 26-27 Nov, p. 201(1997).

El-Sweify, F.H, El-Sweify, A.H.H., Abd El-Razek, A.M.: "Multielements determination samples of some organs of flax, field soil and irrigation water using neutron activation analysis". Arab J. Nucl. Sci. Appl. 30, 199 (1997).

El-Sweify, F.H., Hammad, F., Abd El-Razek. A.M.: Analysis of trace elements in liquid samples of different origans using CNAA. Al-Azhar Bulletin of Sci., Proc., Cairo Egypt, $5^{\text {th }}$. Int. Sci., Conf., 2527 March, P. 15 (2003).

El-Sweify, F.H., Metwally, E., Abdel-Khalik, H.: Analysis of pencil graphite samples of various origins using instrumental neutron activation analysis. J. Radiochim. Acta 96, 63(2008).

Fatma H. El-Sweify, Mahmoud A. El-Amir, Mohamed Mostafa, Hala E. Ramadan, and Ghada M. Rashad, A.M.: "Simultaneous multi-element determination in different seed samples of Dodonaea viscosa hopseed using instrument neutron activation analysis" J. Radiochim. Acta, P. 104(3): 211 220(2016).

Giannossi, M. L., Mongelli, G., Tateo, F., \& Summa, V. "Mineralogical and morphological investigation of kidney stones of a Mediterranean region (Basilicata, Italy)". Journal of X-Ray Science and Technology, 20(2), 175-186. (2012).

Golovanova, O., Palchik, N., Maksimova, N., \& In, A. "Comparative characterization of the microelement composition of kidney stones from patients in the Novosibirsk and Omsk regions" (2009)..

Hesse, A., Tiselius, H. G., Siener, R., \& Hoppe, B. "Urinary stones: diagnosis, treatment, and prevention of recurrence". 3rd revised and enlarged edition. Karger: Basel (2009).

J.C.Bailar, H.J.Emeleus, R.Nyholm, A.F Trotman - Dickenoon "Comprehensive Inorganic Chemistry", vol.4. Lanthanides. (C Pergamon (1973)

Jute (Corchorus capsularis) seed samples. Arab, J. Nucl. Sci. Appl. 37(1), 53 (2004).

N,N.Greenwood and A.Earnshaw " The chemistry of the elements. Butterworth heincmann, Great Britain. November (1997).

Pearle. M. S, Yair Lotan, "Urinary Lithiasis: Etiology, epidemiology, and pathogenesis in: CampbellWalsh urology", 10thed. (2012).

Pourmand, G., \& Pourmand, B. "Epidemiology of stone disease in Iran". In urolithiasis (Vol. 85-87). London: Springer (2012). 
Safarinejad, M. R. "Adult urolithiasis in a population-based study in Iran: prevalence, incidence, and associated risk factors". Urological Research, 35(2), 73-82 (2007).

Shokouhi, B., Gasemi, K., \& Norizadeh, E. "Chemical composition and epidemiological risk factors of urolithiasis in Ardabil Iran". Research Journal of Biological Sciences, 3(6), 620-626 (2008).

Stamatelou, K. K., Francis, M. E., Jones, C. A., Nyberg, L. M., \& Curhan, G. C. "Time trends in reported prevalence of kidney stones in the United States": 1976-19941. Kidney International, 63(5), 1817-1823. (2003).

Tahra El sayed Mohammed Salim., "Studies and application on Neutron Capture $(\mathrm{n}, \gamma)$ using $\mathrm{K}_{0^{-}}$ Method ", physics Department, Women's college for Arts, Science and Education, Ain Shams Univ. (2010)

Talham, D. R., Backov, R., Benitez, I. O., Sharbaugh, D. M., Whipps, S., \& Khan, S. R. "Role of lipids in urinary stones: Studies of calcium oxalate precipitation at phospholipid Langmuir monolayers. Langmui"r, 22(6), 2450-2456. https://doi.org/10.1021/la052503u.

Tölgyesy, J.; et al. (1971).

Tur, J., Prieto, R., \& Grases, F. "An animal model to study the effects of diet on risk factors of calcium stone formation". Scandinavian Journal of Urology and Nephrology, 25(4), 311-314. (1991).

Wijayarathna, K. S. N., \& Abeygunasekera, A. M. "Pathogenesis of nephrolithiasis. Sri Lanka" Journal of Surgery, 31(3), 28-32. https://doi.org/10.1016/j.juro.2012. 11.069. (2013).

Zarasvandi, A., Heidari, M., Sadeghi, M., \& Mousapoor, E. "Major and trace element composition of urinary stones, Khuzestan province, Southwest, Iran". Journal of Geochemical Exploration, 131, 5258. (2013). 


\section{الملخص باللغة العربية}

حول إستخدام تقتية التحليل التششيطي النيتروني الآلي لتحليل بعض عينات الحصوات الكلوية للإنسان

ريهام عاطف ،فاطمة السويفي' ، محمد على'، وفاء حجازي

أ_قسم الكيمياء النوويهـ شعبة انتاج النظائر المشعة (هيئة الطاقة الذرية) ص.ب 09 مب القاهرة ـ مصر بـ كلية البنات للآداب و العلوم و التربية - جامعة عين شمس - القاهرة ـ مصر

- قد إزداد حديثاً معدل مرض الحصوات الكلوية في مصر يرجع ذلك إلى المؤثرات الصناعية و البيئية بالإضافة إلى ذلك فقد يكون للعناصر الضئيلة دوراً في تكوين مثل هذه الحصوات بالرغم من أهميتها في العمليات الحيوية.

- في هذه الدراسة أُستخدمت تقنية التحليل التنشيطي النيتروني الآلي للتحليل العناصري لبعض الحصوات الكلوية التي تم تجميعها من مرضى يعيشون في محافظات مختلفة في مصر. جمعت هذه العينات من مرضى من الرجال و النساء و الأطفال ذوي أعمار مختلفة.

- أجريت التحاليل تحت ظروف مختلفة من أزمنة التشعيع و التبريد وقد قدرت تركيزات عناصر رئيسية وثانوية وكذلك ذات التركيزات الضئيلة. أستخدمت مادة قياسية لقياسات درجة الصوابة ومراقبة الجودة. العناصر المحللة هي: As, Br, Ca, Co, Cr, Cu, Fe, Hf, In, Mg, Mn, Na, Se, Sr, Tb, V Zn وذللك تحت الظروف التجريبية المستخدمة.

- - بعض هذه العناصر أساسية من الناحية الحيوية وبعضها قد يكون خطيراً، في حالة إذا زاد التركيز تم إقتراح بعض الإرتباطات بين تركيز هذه العناصر وكلِ من الجنس والعمر ومكان الإقامة. كما تم إستنباط نوع الحصوات من نتائج تركيز بعض العناصر. 